\title{
10. MAJOR ELEMENT AND OXYGEN ISOTOPE STUDIES OF INTERSTITIAL WATERS: ODP LEG 1131
}

\author{
Per Kristian Egeberg, ${ }^{2}$ Per Aagaard, ${ }^{2}$ and P. Craig Smalley ${ }^{3}$
}

\begin{abstract}
Variations in the distribution of major elements and stable oxygen isotopes in ODP Leg 113 pore water are not related to lithology and thus appear to be controlled by minor constituents. Petrographic observations and geochemical considerations indicate that alteration of calc-alkalic volcanic material dispersed in the sediment is an important process. A diagenetic reaction is constructed that involves transformation of volcanic glass into smectite, zeolite (represented by phillipsite), chert, and iron sulfide. Mass balance calculations reveal that alteration of less than $10 \%$ (volume) of volcanogenic material may account for the observed depletion of magnesium, potassium, and ${ }^{18} \mathrm{O}$ and enrichment of calcium. Alteration of this amount of volcanic glass produces less than $4 \%$ (volume) of smectite and zeolite. Hence, mass balance is obtained without having to invoke unreasonable large amounts of volcanic matter or interactions between seawater and basement.
\end{abstract}

\section{INTRODUCTION}

Interstitial water extracted from cores from 12 holes provide complete vertical profiles of interstitial water constituents at 7 sites.

Here we report both the shipboard geochemical results on major ion composition and results obtained in our shore-based laboratory. Lithostratigraphy, sedimentology, clay mineralogy, and biostratigraphy are given in other chapters of this volume, thus for each site we only give a condensed description of the observations relevant to interstitial water chemical studies (see below).

We wish to focus our discussion on the chemistry of the alkaline earth metals. Usually, in pelagic and hemipelagic deposits, the concentrations of calcium increase and the concentration of magnesium decrease with increasing depth of burial (Gieskes, 1973; Gieskes and Lawrence, 1976; McDuff, 1981; Gieskes et al., 1984; Baker, 1986). Commonly the changes in calcium and magnesium concentrations with depth can be linearly correlated with $\Delta \mathrm{Ca} / \Delta \mathrm{Mg}$ slopes ranging from -0.2 (Gieskes et al., 1978) to -3 (Gieskes and Lawrence, 1976). Based on a compilation of data from several DSDP-cruises Baker (1986) calculated an average slope of -0.45 . He attributed different slopes to the nature of the basement, low slopes being associated with siliceous basement and steep slopes with basaltic basement. He argued that the alteration of continental (siliceous) basement releases more calcium per mole of magnesium taken up than does alteration of oceanic (basaltic) basement. At sites with pronounced nonlinear concentration profiles, diagenetic reactions taking place in the sediment column must also be influencing interstitial water elemental concentrations.

Based on the 1:1 relationship between $\Delta \mathrm{Ca}$ and $\Delta \mathrm{Mg}$ and the presence of authigenic dolomite, Manheim (1976) favored dolomitization as the mechanism responsible for the pore water gradients in dissolved alkaline earths. This mechanism preserves alkalinity. Mackenzie and Garrels (1966) proposed that reactions involving reconstitution of clays, termed "reverse weathering," are responsible for much of the removal of magnesium. Such a

\footnotetext{
${ }^{1}$ Barker, P. F., Kennett, J. P., et al., 1990. Proc. ODP, Sci. Results, 113: College Station, TX (Ocean Drilling Program)

2 Department of Geology, P.O. Box 1047, Blindern, N-0316 Oslo 3, Norway.

3 Institute for Energy Technology, P.O. Box 40, Kjeller, N-2007, Norway.
}

process removes alkalinity and produces carbon dioxide. One of the difficulties in accepting this mechanism is the disposal of excess $\mathrm{CO}_{2}$ (Manheim, 1976; Perry et al., 1976). Wollast (1974) avoided this problem by having $\mathrm{CO}_{2}$ react with calcite, this reaction also accounts for the increase in calcium. Alternatively $\mathrm{CO}_{2}$ may be consumed by attacking plagioclase. Drever (1974) demonstrated that ion exchange may account for removal of $8 \%$ of the river-derived magnesium. This process is rapid and virtually completed at the time of deposition and probably does not take any part in the magnesium budget of deep sea sediments. Matter et al., (1975) observed that recrystallization of low Mg-calcite constitutes an additional sink for magnesium. Strontium isotope measurements on dissolved strontium carried out by Hawkesworth and Elderfield (1978) demonstrated that volcanic ash may be very reactive during diagenesis. By combining major element analyses with ${ }^{18} \mathrm{O} /{ }^{16} \mathrm{O}$-measurements, Perry et al., 1976 were able to demonstrate that alteration of calc-alkalic volcanics may serve both as a source of calcium and as a sink for magnesium. The alteration product is believed to be smectite. The alteration reaction conserves alkalinity. Another process proposed for the removal of magnesium from the interstitial water is $\mathrm{Mg}$ Fe exchange in clays driven by sulfidic reduction of ferric iron (Drever, 1971). From experimental studies Heller-Kallai and Rozenson (1978) concluded that direct exchange between $\mathrm{Fe}$ and $\mathrm{Mg}$ seems improbable.

\section{ANALYTICAL METHODS}

The interstitial water was extracted on board using a stainless steel hydraulic press and the procedures described by Manheim and Sayles (1974). Shipboard analyses of chloride, calcium, magnesium, dissolved silicon, and alkalinity were carried out according to the methods described by Gieskes (1974). Potassium and sulfate were determined by ion chromatography and onshore analyses of strontium were carried out by atomic absorption spectroscopy after having adjusted the samples to 4000 ppm La ${ }^{3+}$ with $\mathrm{LaCl}_{3}$ (Gieskes, 1974). The $\delta^{18} \mathrm{O}$ values of pore waters were determined using the $\mathrm{CO}_{2}$ equilibration method. Isotope analyses were performed on a Finnigan MAT 251 mass spectrometer. The results are reproducible to $\pm 0.2 \% 0$ ( 2 sigma).

\section{SITE DESCRIPTION}

\section{Site 689 (Maud Rise)}

The site is located near the crest of Maud Rise (Fig. 1) at a water depth of $2080 \mathrm{~m}$. Whole round samples for squeezing 


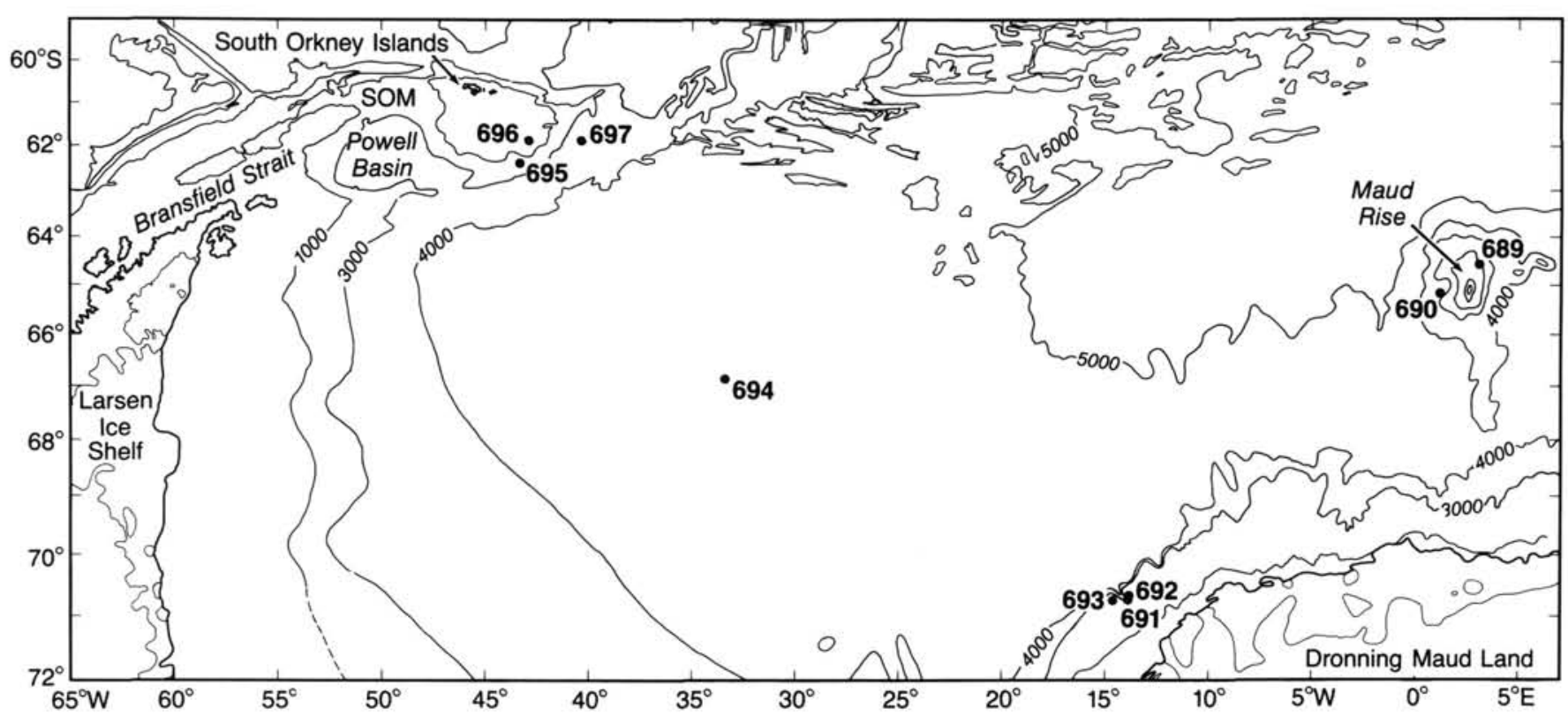

Figure 1. ODP Leg 113 Site locations. SOM = South Orkney microcontinent.

were taken from cores collected from Hole 689B drilled to 297.3 mbsf, terminated approximately $40 \mathrm{~m}$ above basement. The recovered sediments are pelagic, biogenic in origin, and range in age from Campanian/Maestrichtian to Pleistocene. The sequence has been divided into three lithostratigraphic units based upon compositional differences and diagenetic maturity.

Unit I (0-31.0 mbsf; late Miocene to Pleistocene) consists predominantly of diatom ooze with varying amounts of other biosiliceous components.

Unit II (31.0-149.1 mbsf; late Eocene to late Miocene) consists of a mixture of biosiliceous and calcareous oozes. The calcareous component (mainly nannofossils) increases downward.

Unit III (149.1-297.3 mbsf; Campanian? to late Eocene) consists of nannofossil ooze and chalk.

Zeolites, chert, and altered volcanic ash-bearing layers are minor components. The clay size fraction (Barker et al., 1988) is dominated by smectite and illite down to $110 \mathrm{mbsf}$ and by smectite below 110 mbsf.

\section{Site 690 (Maud Rise)}

This site is situated on the southwestern flank of Maud Rise in $2914 \mathrm{~m}$ of water. Whole round samples for squeezing were taken from cores from Hole 690B drilled to 213.4 mbsf and from Hole $690 \mathrm{C}$, terminated in basaltic rock at $321.2 \mathrm{mbsf}$. The sediments range in age from Late Cretaceous to Pleistocene. The sequence is mainly biogenic, but terrigenous and volcanic detritus become a significant component around $140 \mathrm{mbsf}$. Thin, rare chert layers were recovered. The sequence has been divided into six lithostratigraphic units.

Unit I (0-24.4 mbsf; late Miocene to Pleistocene) consists of $2.1 \mathrm{~m}$ of foraminiferal ooze overlying diatom ooze with varying amounts of other biosiliceous components.

Unit II (24.4-92.9 mbsf; early Oligocene to late Miocene) consists of pure and mixed biogenic siliceous and calcareous oozes.
Unit III (92.9-137.8 mbsf; late Paleocene to early Oligocene) consists of foraminiferal oozes.

Unit IV (137.8-281.1 mbsf; late Maestrichtian to late Paleocene) consists of nannofossil oozes and chalks with a terrigenous component of $10 \%-15 \%$.

Unit V (281.1-317.0 mbsf; late Campanian(?) to late Maestrichtian) consists predominantly of terrigenous material (including volcanic glass) and contains calcareous ooze and chalk.

Unit VI (317.0-321.1 mbsf) consists of $1.71 \mathrm{~m}$ of amygdaloidal pyroxene-olivine basalts.

The clay size fraction (Barker, Kennett, et al., 1988) is dominated by smectite except in the upper 90 mbsf where illite is equal to or more common. Chlorite + kaolinite is present down to $213 \mathrm{mbsf}$. Zeolites are present at $230-260 \mathrm{mbsf}$.

\section{Site 693 (Dronning Maud Land margin)}

Site 693 lies on a mid-slope bench of the Weddell Sea margin of East Antarctica in $2359 \mathrm{~m}$ of water. Whole round samples for squeezing were taken from cores from Hole 693A drilled to $483.9 \mathrm{mbsf}$ and from Hole 693B drilled to $403.1 \mathrm{mbsf}$. The seismic records indicate the presence of at least another $1000 \mathrm{~m}$ of sediments below our maximum penetration. The sediments range in age from Lower Cretaceous to Pleistocene. The sequence has been divided into seven lithostratigraphic units.

Unit I (0-12.2 mbsf; Pleistocene) consists of foraminiferbearing clayey mud.

Unit II (12.2-31.4 mbsf; late Pliocene to Pleistocene) consists of clayey mud.

Unit III (31.4-325.8 mbsf; late Oligocene to late Pliocene) consists of diatom mud and diatom-bearing silty to clayey mud.

Unit IV (325.8-345.1 mbsf; late Oligocene) consists of alternating diatomaceous mud and ooze and minor muddy nannofossil ooze and nannofossil-bearing clayey mud.

Unit V (345.1-397.8 mbsf; Oligocene) consists predominantly of diatomaceous mud and silt. 
Unit VI (397.8-409.0 mbsf; Cretaceous-?Albian to Santonian) consists of radiolarian diatomite.

Unit VII (409.0-483.9 mbsf; Early Cretaceous-Albian) consists of terrigenous claystones and mudstones with organic rich beds.

The clay size fraction (Barker, Kennett, et al., 1988) is dominated by illite down to $403.1 \mathrm{mbsf}$ and by smectite from 403.1 mbsf to 483.9 mbsf. Kaolinite and chlorite are present in most samples above 403.1 mbsf. Volcanic glass and opaque minerals are present throughout as minor components.

\section{Site 694 (Weddell abyssal plain)}

Site 694 is situated on the northern part of the Weddell abyssal plain in $4653 \mathrm{~m}$ of water. Whole round samples for squeezing were collected from cores from Hole 694B, drilled to 179.2 mbsf and from Hole $694 \mathrm{C}$ terminated at $391.3 \mathrm{mbsf}$. The seismic records indicate the presence of more than $1000 \mathrm{~m}$ of sediment below our total depth. The recovered sediments range in age from middle Miocene to Pleistocene and are mostly terrigenous with a minor biosilicious component. Four lithostratigraphic units were defined.

Unit I (0-21.1 mbsf; early Pliocene to Pleistocene) consists of terrigenous clay and clayey mud with minor silt and diatom-bearing clayey mud.

Unit II (21.1-111.5 mbsf; early Pliocene?) consists predominantly of well- to moderately-well-sorted lithic and quartz sands.

Unit III (111.5-304.3 mbsf; middle Miocene? to early Pliocene?) consists of hemipelagic sediments and turbidites, graded silt sequences with some diatoms, and diatombearing silty and clayey muds with interbedded silts and sandy muds.

Unit IV (304.3-391.3 mbsf; middle Miocene) consists of diatom-bearing and diatomaceous claystones, with silt near the base.

The clay size fraction (Barker, Kennett, et al., 1988) is dominated by illite and chlorite. The proportion of chlorite increases downward while the abundance of kaolinite and smectite generally decrease toward the base of the sequence. Opaque minerals are abundant.

\section{Site 695 (South Orkney microcontinent)}

Site 695 is located on the southeastern edge of the South Orkney microcontinent in $1305 \mathrm{~m}$ of water. Whole round samples for squeezing were sampled from cores recovered from Hole $695 \mathrm{~A}$ drilled to $352.1 \mathrm{mbsf}$. The seismic records indicate the presence of at least another $1500 \mathrm{~m}$ of sediment below. The sediments are biosiliceous hemipelagic deposits and range in age from Miocene/Pliocene to Pleistocene. Three lithostratigraphic units were defined.

Unit I (0-190.0 mbsf; Pleistocene to early Pliocene) consists of diatom-bearing silty and clayey muds, muddy diatom ooze, diatom ooze, and diatom silty muds.

Unit II (190.0-306.9 mbsf; early Pliocene) consists of diatom-bearing silty and clayey muds.

Unit III (306.9-341.1 mbsf; early Pliocene to late Miocene) consists of silty mud with $0 \%-10 \%$ diatoms.

The clay size fraction (Barker, Kennett, et al., 1988) is dominated by illite and chlorite, the proportion of kaolinite decreases downhole, while the relative abundance of smectite varies randomly. Volcanic glass and rock fragments are observed throughout.

\section{Site 696 (South Orkney microcontinent)}

Site 696 is situated on the southeastern margin of the South Orkney microcontinent, under $650 \mathrm{~m}$ of water. Whole round samples for squeezing were taken from cores from Hole 696A drilled to 106.0 mbsf and from Hole 696B terminated at 647.0 mbsf. The seismic records indicate total sediment thickness of at least $1200 \mathrm{~m}$. The sediments are terrigenous, hemipelagic, and pelagic and range in age from middle/upper Eocene to Pleistocene. The sequence has been divided into seven lithostratigraphic units.

Unit I (0-64.2 mbsf; Pleistocene to early late Pliocene) consists primarily of diatomaceous muds and oozes.

Unit II (64.2-124.8 mbsf; early late Pliocene to early Pliocene) consists mainly of diatom-bearing silty and clayey mud.

Unit III (124.8-211.8 mbsf; early Pliocene to late Miocene) consists predominantly of silty and clayey mud, with less important diatom-bearing clayey mud.

Unit IV (211.8-260.1 mbsf; late Miocene) consists of diatom ooze and muddy diatom ooze.

Unit V (260.1-269.7 mbsf; late Miocene) consists of coarsegrained sand.

Unit VI (269.7-529.8 mbsf; late Miocene to middle Miocene) consists of diatom ooze, mud-bearing diatom ooze, diatomite, and mud-bearing diatomite.

Unit VII (529.8-645.6 mbsf; middle? to late Eocene) consists of sandy mudstone, claystone, clayey mudstone, silty mudstone, and barren glauconitic silty mudstone.

The clay size fraction (Barker, Kennett, et al., 1988) is dominated by chlorite and illite down to $500 \mathrm{mbsf}$ and by smectite from $500 \mathrm{mbsf}$ to $645.6 \mathrm{mbsf}$. Volcanic ash is present as layers throughout most of the sequence and is particularly abundant between 269.7 and $529.8 \mathrm{mbsf}$; finely dispersed glass constitutes $4 \%-8 \%$ of the sediment (smear slide estimates).

\section{Site 697 (South Orkney microcontinent-Jane Basin)}

Site 697 is located in Jane Basin under $3484 \mathrm{~m}$ of water. Whole round samples for squeezing were taken from cores from Hole 697A, drilled to $28.1 \mathrm{mbsf}$ and from Hole 697B, terminated at $304.9 \mathrm{mbsf}$. The seismic records indicate a total sediment thickness of at least $800 \mathrm{~m}$. The recovered sediments are hemipelagic with a minor biosiliceous component, and range in age from Pliocene to Pleistocene. Two lithostratigraphic units were defined.

Unit I (0-293.0 mbsf; Pliocene to Pleistocene) consists of silty mud, diatom-bearing silty mud, clayey mud, and diatom-bearing clayey mud.

Unit II (293.0-322.9 mbsf; early Pliocene) consists of silty and clayey mud.

The clay size fraction (Barker, Kennett, et al., 1988) is dominated by illite and chlorite; kaolinite and smectite are present at most depths. Volcanic ash is abundant.

\section{RESULTS}

The concentration of dissolved constituents is listed in Tables 1-7 and shown graphically in Figures 2-8. Only minor variations in the concentration of chloride are observed. Generally, although to a different extent at different sites, the pore waters are depleted in magnesium, potassium, and sulfate and enriched in calcium, strontium, dissolved silicon, and alkalinity relative to seawater of the same chlorinity. With the exception of the up- 
Table 1. Interstitial water concentration data, Site 689.

\begin{tabular}{lrccccccccrr}
\hline $\begin{array}{l}\text { Core, section } \\
\text { interval }(\mathrm{cm})\end{array}$ & $\begin{array}{c}\mathrm{Depth} \\
(\mathrm{mbsf})\end{array}$ & $\mathrm{pH}$ & $\begin{array}{c}\mathrm{Ca}^{2}+ \\
(\mathrm{mmol})\end{array}$ & $\begin{array}{c}\mathrm{Mg}^{2+} \\
(\mathrm{mmol})\end{array}$ & $\begin{array}{c}\mathrm{Sr}^{2}+ \\
(\mu \mathrm{mol})\end{array}$ & $\begin{array}{c}\mathrm{K}+ \\
(\mathrm{mmol})\end{array}$ & $\begin{array}{c}\mathrm{Cl}- \\
(\mathrm{mmol})\end{array}$ & $\begin{array}{c}\mathrm{SO}^{2-} \\
(\mathrm{mmol})\end{array}$ & $\begin{array}{c}\text { Alk. } \\
(\mathrm{meq} / \mathrm{L})\end{array}$ & $\begin{array}{c}\mathrm{SiO}_{2} \\
(\mu \mathrm{mol})\end{array}$ & $\begin{array}{r}\delta 18_{0} \\
(\% 0)^{\mathrm{a}}\end{array}$ \\
\hline $1 \mathrm{H}-2,135-142$ & 2.85 & & 11.39 & 53.71 & 90 & 12.3 & 554 & 29.5 & & 994 & 1.2 \\
$1 \mathrm{H}-3,78-85$ & 3.78 & & 11.54 & 53.72 & & 12.0 & 551 & 29.2 & & 1062 & \\
$1 \mathrm{H}-3,145-150$ & 4.45 & 7.82 & 11.58 & 52.79 & 101 & 11.6 & 547 & 29.5 & 2.91 & 815 & 0.2 \\
$2 \mathrm{H}-2,66-70$ & 7.46 & & 11.35 & 53.81 & 104 & 11.4 & 551 & 28.9 & & 1196 & 0.7 \\
$3 \mathrm{H}-4,120-125$ & 20.50 & 7.65 & 11.92 & 52.78 & 111 & 11.1 & 556 & 28.0 & 3.19 & 819 & -0.1 \\
$6 \mathrm{H}-4,120-125$ & 49.00 & 7.66 & 12.67 & 51.71 & 128 & 12.5 & 560 & 28.3 & 3.15 & 796 & -1.2 \\
$9 \mathrm{H}-4,120-125$ & 77.80 & 7.31 & 13.49 & 50.97 & 147 & 11.4 & 559 & 26.7 & 2.90 & 808 & -1.7 \\
$12 \mathrm{H}-4,115-125$ & 106.65 & 7.66 & 14.77 & 49.56 & 156 & 10.2 & 558 & 26.7 & 3.10 & 787 & -1.2 \\
$15 \mathrm{H}-4,115-125$ & 135.55 & 7.78 & 16.11 & 50.35 & 147 & 10.5 & 581 & 28.0 & 3.06 & 774 & -1.5 \\
$18 \mathrm{H}-4,115-125$ & 164.45 & 7.81 & 16.58 & 48.11 & 194 & 10.7 & 558 & 25.7 & 3.10 & 608 & -1.4 \\
$22 \mathrm{X}-4,115-125$ & 203.15 & 7.48 & 17.97 & 47.21 & 200 & 9.9 & 559 & 26.0 & 2.43 & 606 & -0.9 \\
$25 \mathrm{X}-4,115-125$ & 232.25 & 7.54 & 18.67 & 46.67 & 209 & 10.2 & 558 & 26.8 & 2.35 & 580 & -0.9 \\
$28 \mathrm{X}-3,115-125$ & 259.72 & 7.57 & 20.86 & 45.33 & 219 & 9.0 & 560 & 26.3 & 2.06 & 570 & -0.4 \\
\hline
\end{tabular}

a Relative to SMOW.

Table 2. Interstitial water concentration data, Site 690.

\begin{tabular}{|c|c|c|c|c|c|c|c|c|c|c|c|}
\hline $\begin{array}{l}\text { Core, section } \\
\text { interval }(\mathrm{cm})\end{array}$ & $\begin{array}{l}\text { Depth } \\
\text { (mbsf) }\end{array}$ & $\mathrm{pH}$ & $\begin{array}{l}\mathrm{Ca}^{2+} \\
(\mathrm{mmol})\end{array}$ & $\begin{array}{l}\mathrm{Mg}^{2+} \\
(\mathrm{mmol})\end{array}$ & $\begin{array}{l}\mathrm{Sr}^{2+} \\
(\mu \mathrm{mol})\end{array}$ & $\underset{(\mathrm{mmol})}{\mathrm{K}+}$ & $\begin{array}{c}\mathrm{Cl}- \\
(\mathrm{mmol})\end{array}$ & $\begin{array}{l}\mathrm{SO}^{2-} \\
(\mathrm{mmol})\end{array}$ & $\underset{(\mathrm{meq} / \mathrm{L})}{\mathrm{Alk}}$ & $\begin{array}{c}\mathrm{SiO}_{2} \\
(\mu \mathrm{mol})\end{array}$ & $\begin{array}{l}\delta 18_{0} \\
(\% 0)^{\mathrm{a}}\end{array}$ \\
\hline $2 \mathrm{H}-3,145-150$ & 6.55 & 7.70 & 10.80 & 54.13 & 99 & 12.8 & 547 & 29.2 & 2.84 & 738 & -0.5 \\
\hline $3 \mathrm{H}-4,120-125$ & 17.40 & 7.52 & 10.83 & 54.10 & 104 & 12.9 & 556 & 29.2 & 2.93 & 1126 & -0.5 \\
\hline $6 \mathrm{H}-4,120-125$ & 46.50 & 7.96 & 11.59 & 54.36 & 109 & 12.4 & 553 & 27.6 & 3.11 & 707 & -1.1 \\
\hline $9 \mathrm{H}-4,120-125$ & 75.50 & 7.81 & 11.69 & 52.70 & 138 & 12.0 & 553 & 26.5 & 3.75 & 721 & -1.4 \\
\hline $12 \mathrm{H}-5,120-125$ & 106.00 & 7.69 & 12.77 & 54.15 & 127 & 11.5 & 550 & 24.7 & 3.79 & 394 & -2.1 \\
\hline $16 \mathrm{H}-4,120-125$ & 143.50 & 7.90 & 13.01 & 51.38 & 147 & 10.6 & 545 & 22.9 & 4.04 & 238 & -1.6 \\
\hline $19 \mathrm{H}-4,115-125$ & 172.55 & 7.83 & 12.83 & 48.76 & 146 & 10.0 & 550 & 22.3 & 3.82 & 140 & -2.1 \\
\hline $22 \mathrm{H}-3,120-125$ & 189.40 & 7.77 & 13.00 & 49.00 & 146 & 11.1 & 550 & 24.6 & 3.84 & 193 & -2.3 \\
\hline $25 \mathrm{H}-4,115-125$ & 209.85 & 7.74 & 13.00 & 48.37 & 155 & 10.6 & 547 & 22.7 & 3.87 & 225 & -2.5 \\
\hline $11 X-3,115-125$ & 208.35 & 7.95 & 12.91 & 50.17 & 127 & 10.0 & 545 & 21.9 & 3.27 & 278 & -1.5 \\
\hline $14 X-4,115-125$ & 238.85 & 7.75 & 13.10 & 48.49 & 124 & 9.8 & 548 & 20.1 & 3.87 & 365 & -1.5 \\
\hline $17 X-2,115-125$ & 264.45 & 7.63 & 13.13 & 50.76 & 160 & 9.6 & 559 & 22.8 & 3.47 & 642 & -1.5 \\
\hline $20 X-4,115-125$ & 296.45 & 7.86 & 16.12 & 51.27 & 159 & 9.2 & 529 & 21.0 & 3.10 & 619 & -1.7 \\
\hline
\end{tabular}

a Relative to SMOW.

Table 3. Interstitial water concentration data, Site 693.

\begin{tabular}{|c|c|c|c|c|c|c|c|c|c|c|c|}
\hline $\begin{array}{l}\text { Core, section } \\
\text { interval }(\mathrm{cm})\end{array}$ & $\begin{array}{l}\text { Depth } \\
\text { (mbsf) }\end{array}$ & $\mathrm{pH}$ & $\begin{array}{l}\mathrm{Ca}^{2+} \\
(\mathrm{mmol})\end{array}$ & $\begin{array}{l}\mathrm{Mg}^{2+} \\
(\mathrm{mmol})\end{array}$ & $\begin{array}{l}\mathrm{Sr}^{2+} \\
(\mu \mathrm{mol})\end{array}$ & $\underset{(\mathrm{mmol})}{\mathrm{K}+}$ & $\begin{array}{c}\mathrm{Cl}- \\
(\mathrm{mmol})\end{array}$ & $\begin{array}{l}\mathrm{SO}^{2-} \\
(\mathrm{mmol})\end{array}$ & $\begin{array}{c}\text { Alk. } \\
(\mathrm{meq} / \mathrm{L})\end{array}$ & $\underset{(\mu \mathrm{mol})}{\mathrm{SiO}_{2}}$ & $\begin{array}{l}\delta 18_{0} \\
(\% 0)^{\mathrm{a}}\end{array}$ \\
\hline $2 \mathrm{R}-4,145-150$ & 8.45 & 7.90 & 10.65 & 50.73 & 76 & 11.8 & 577 & 28.6 & 3.90 & 433 & -0.5 \\
\hline $4 \mathrm{R}-3,120-125$ & 26.10 & 7.83 & 10.59 & 50.42 & 85 & 11.8 & 577 & 27.7 & 3.56 & 639 & -0.7 \\
\hline $6 \mathrm{R}-4,120-125$ & 46.80 & 7.76 & 14.1 & 46.98 & 94 & 11.3 & 579 & 27.8 & 3.49 & 803 & -1.1 \\
\hline $9 \mathrm{R}-4,120-125$ & 75.80 & 8.10 & 15.37 & 44.77 & 94 & 10.1 & 569 & 25.1 & 3.75 & 1053 & -0.7 \\
\hline $12 \mathrm{R}-4,120-125$ & 104.60 & 8.15 & 17.07 & 43.08 & 85 & 8.3 & 571 & 25.6 & 3.88 & 880 & -1.5 \\
\hline $18 \mathrm{R}-3,120-125$ & 161.10 & 8.19 & 18.00 & 41.87 & 103 & 7.8 & 579 & 22.7 & 3.36 & 904 & -2.3 \\
\hline $25 \mathrm{R}-2,120-125$ & 227.30 & 8.07 & 17.73 & 38.89 & 124 & 7.4 & 581 & 20.3 & 3.32 & 1000 & -2.8 \\
\hline $2 X-3,145-150$ & 238.25 & 8.03 & 17.16 & 40.3 & 135 & 7.7 & 563 & 21.3 & 2.79 & 1100 & -3.2 \\
\hline $28 \mathrm{R}-3,120-125$ & 257.80 & 8.10 & 17.12 & 38.97 & 127 & 7.7 & 572 & 19.3 & 3.14 & 933 & -1.1 \\
\hline $6 X-4,145-150$ & 273.75 & 7.98 & 17.42 & 37.43 & 136 & 8.0 & 561 & 18.6 & 2.99 & 1370 & -1.3 \\
\hline $9 \times-3,120-125$ & 301.00 & 8.24 & 17.10 & 37.87 & 134 & 7.8 & 561 & 19.1 & 3.19 & 1200 & -2.9 \\
\hline $12 X-3,120-125$ & 330.00 & 7.76 & 16.72 & 37.83 & 154 & 7.5 & 562 & 17.8 & 2.27 & 1300 & -2.6 \\
\hline $15 X-2,140-150$ & 357.60 & 8.34 & 16.71 & 38.06 & 138 & 7.8 & 563 & 18.1 & 3.45 & 1035 & -1.2 \\
\hline $40 \mathrm{R}-2,138-142$ & 371.78 & 8.16 & 16.98 & 35.87 & 147 & 7.8 & 557 & 17.2 & 3.02 & 1020 & -1.8 \\
\hline $17 X-1,145-150$ & 375.45 & 8.28 & 16.85 & 36.94 & 173 & 7.8 & 567 & 17.2 & 2.83 & 950 & -3.8 \\
\hline $19 X-2,140-150$ & 396.30 & 8.20 & 17.39 & 36.77 & 166 & 7.3 & 565 & 16.9 & 3.55 & 995 & -3.1 \\
\hline $48 \mathrm{R}-2,120-125$ & 448.10 & 8.16 & 16.15 & 27.27 & 168 & 6.3 & 519 & 16.0 & 2.83 & 394 & \\
\hline
\end{tabular}

a Relative to SMOW.

per three samples at Site 689, which were collected from disturbed sections, only negative $\delta^{18} \mathrm{O}$ values were observed. Pore water $\delta^{18} \mathrm{O}$ values generally become depleted with depth, but considerable scatter is present. In some instances (e.g., Cores 113-696B-3R-3, 113-696B-26R-2, and 113-696B-34R-1), anomalous $\delta^{18} \mathrm{O}$ values are associated with anomalous sulfate concentrations, thus some contamination by seawater may have taken place. However, in most cases the scatter in the oxygen isotope data is not related to fluctuations in the concentration of any of the other dissolved constituents. The fluctuations are much larger than what one would expect from the reproducibility of the method $( \pm 0.2 \%)$ and are probably partly due to analytical difficulties. We believe the data represent the general trends, but do not trust them sufficiently to go into any detailed discussion.

The profiles composed of data from cores from more than one hole display excellent continuity between the two data sets. Because of their uniformity, data from all sites will be discussed collectively. 
Table 4. Interstitial water concentration data, Site 694.

\begin{tabular}{|c|c|c|c|c|c|c|c|c|c|c|c|}
\hline $\begin{array}{l}\text { Core, section } \\
\text { interval }(\mathrm{cm})\end{array}$ & $\begin{array}{l}\text { Depth } \\
\text { (mbsf) }\end{array}$ & $\mathrm{pH}$ & $\begin{array}{l}\mathrm{Ca}^{2+} \\
(\mathrm{mmol})\end{array}$ & $\begin{array}{l}\mathrm{Mg}^{2+} \\
(\mathrm{mmol})\end{array}$ & $\begin{array}{l}\mathrm{Sr}^{2+} \\
(\mu \mathrm{mol})\end{array}$ & $\underset{(\mathrm{mmol})}{\mathrm{K}+}$ & $\underset{(\mathrm{mmol})}{\mathrm{Cl}-}$ & $\begin{array}{l}\mathrm{SO}^{2-} \\
(\mathrm{mmol})\end{array}$ & $\begin{array}{c}\text { Alk. } \\
(\mathrm{meq} / \mathrm{L})\end{array}$ & $\underset{(\mu \mathrm{mol})}{\mathrm{SiO}_{2}}$ & $\begin{array}{l}\delta 18_{0} \\
(\% 0)^{\mathrm{a}}\end{array}$ \\
\hline $1 \mathrm{H}-2,145-150$ & 2.95 & 7.91 & 10.25 & 52.52 & 83 & 10.4 & 553 & 28.1 & 3.10 & 165 & -0.3 \\
\hline $3 \mathrm{H}-2,120-125$ & 17.79 & 7.79 & 11.13 & 52.14 & 92 & 9.0 & 559 & 28.3 & 2.75 & 360 & -0.8 \\
\hline $22 X-1,145-150$ & 151.85 & 7.91 & 18.19 & 44.54 & 93 & 5.7 & 570 & 23.0 & 3.07 & 880 & -0.6 \\
\hline $24 X-2,120-125$ & 172.30 & 7.99 & 18.47 & 43.30 & 93 & 4.7 & 565 & 21.7 & 3.40 & 910 & -1.1 \\
\hline $5 X-1,145-150$ & 210.35 & 8.38 & 20.17 & 43.47 & 102 & 5.2 & 570 & 20.6 & 3.15 & 720 & -1.8 \\
\hline $14 X-4,115-125$ & 300.35 & 8.44 & 22.47 & 39.82 & 102 & 3.8 & 572 & 18.3 & 3.00 & 860 & -2.6 \\
\hline $19 X-2,145-150$ & 345.95 & 8.20 & 24.40 & 36.03 & 111 & 3.3 & 567 & 16.2 & 3.55 & 1010 & -1.1 \\
\hline $22 \times-2,115-125$ & 374.65 & 8.20 & 25.49 & 35.08 & 115 & 3.8 & 568 & 15.4 & 3.01 & 1190 & -2.7 \\
\hline
\end{tabular}

a Relative to SMOW.

Table 5. Interstitial water concentration data, Site 695.

\begin{tabular}{|c|c|c|c|c|c|c|c|c|c|c|c|}
\hline $\begin{array}{l}\text { Core, section } \\
\text { interval }(\mathrm{cm})\end{array}$ & $\begin{array}{l}\text { Depth } \\
\text { (mbsf) }\end{array}$ & $\mathrm{pH}$ & $\begin{array}{l}\mathrm{Ca}^{2+} \\
(\mathrm{mmol})\end{array}$ & $\begin{array}{l}\mathrm{Mg}^{2+} \\
(\mathrm{mmol})\end{array}$ & $\begin{array}{l}\mathrm{Sr}^{2+} \\
(\mu \mathrm{mol})\end{array}$ & $\underset{(\mathrm{mmol})}{\mathrm{K}+}$ & $\underset{(\mathrm{mmol})}{\mathrm{Cl}-}$ & $\begin{array}{l}\mathrm{SO}^{2-} \\
(\mathrm{mmol})\end{array}$ & $\begin{array}{c}\text { Alk. } \\
(\mathrm{meq} / \mathrm{L})\end{array}$ & $\underset{(\mu \mathrm{mol})}{\mathrm{SiO}_{2}}$ & $\begin{array}{l}\delta 18_{0} \\
(\% 0)^{\mathrm{a}}\end{array}$ \\
\hline $1 \mathrm{H}-3,145-150$ & 4.45 & 7.86 & 10.66 & 52.15 & 93 & 12.0 & 557 & 29.7 & 3.72 & 527 & -1.2 \\
\hline $3 \mathrm{H}-4,140-145$ & 18.10 & 7.92 & 10.75 & 52.02 & 89 & 11.5 & 561 & 29.4 & 3.78 & 760 & 0.0 \\
\hline $6 \mathrm{H}-3,120-125$ & 45.40 & 8.00 & 10.97 & 51.27 & 90 & 10.3 & 564 & 28.3 & 5.23 & 727 & -1.2 \\
\hline $9 \mathrm{H}-2,120-125$ & 72.70 & 8.00 & 11.39 & 49.56 & 103 & 9.3 & 571 & 24.1 & 7.07 & 875 & -0.9 \\
\hline $12 \mathrm{H}-4,120-125$ & 94.90 & 8.11 & 11.38 & 45.33 & 99 & 8.8 & 562 & 21.9 & 7.95 & 1035 & -1.5 \\
\hline $15 \mathrm{H}-3,120-125$ & 122.40 & 8.40 & 11.27 & 44.79 & 110 & 7.7 & 568 & 19.0 & 8.14 & 950 & -1.3 \\
\hline $19 \times-4,120-125$ & 153.00 & 7.78 & 11.48 & 42.56 & 115 & 9.2 & 566 & 17.6 & 9.35 & 921 & -1.4 \\
\hline $22 X-3,120-125$ & 180.70 & 7.78 & 11.35 & 39.45 & 118 & 8.0 & 560 & 14.1 & 9.62 & 975 & -1.8 \\
\hline $25 \mathrm{X}-3,120-125$ & 200.10 & 7.84 & 11.46 & 38.66 & 120 & 7.8 & 561 & 13.8 & 9.86 & 1117 & -2.1 \\
\hline $29 X-2,115-125$ & 237.35 & 8.17 & 11.86 & 34.06 & 124 & 7.8 & 561 & 9.3 & 10.99 & 1125 & -2.1 \\
\hline $32 X-4,115-125$ & 259.75 & 8.30 & 11.97 & 32.41 & 125 & 7.7 & 565 & 7.7 & 11.10 & 1073 & -1.3 \\
\hline $36 X-2,115-125$ & 295.45 & 7.91 & 12.43 & 30.29 & 131 & 7.4 & 566 & 5.3 & 12.32 & 1108 & -1.8 \\
\hline $39 X-3,115-125$ & 316.15 & 7.75 & 12.53 & 28.68 & 127 & 6.3 & 569 & 1.8 & 12.17 & 1161 & -2.2 \\
\hline $41 X-3,140-150$ & 335.80 & 8.08 & 12.81 & 26.39 & 134 & 5.5 & 571 & 1.2 & 12.37 & 994 & -2.3 \\
\hline
\end{tabular}

a Relative to SMOW.

Table 6. Interstitial water concentration data, Site 696.

\begin{tabular}{|c|c|c|c|c|c|c|c|c|c|c|c|}
\hline $\begin{array}{l}\text { Core, section } \\
\text { interval }(\mathrm{cm})\end{array}$ & $\begin{array}{l}\text { Depth } \\
\text { (mbsf) }\end{array}$ & $\mathrm{pH}$ & $\begin{array}{l}\mathrm{Ca}^{2+} \\
(\mathrm{mmol})\end{array}$ & $\begin{array}{l}\mathrm{Mg}^{2+} \\
(\mathrm{mmol})\end{array}$ & $\begin{array}{l}\mathrm{Sr}^{2+} \\
(\mu \mathrm{mol})\end{array}$ & $\underset{(\mathrm{mmol})}{\mathrm{K}+}$ & $\underset{(\mathrm{mmol})}{\mathrm{Cl}-}$ & $\begin{array}{l}\mathrm{SO}^{2-} \\
(\mathrm{mmol})\end{array}$ & $\begin{array}{c}\text { Alk. } \\
(\mathrm{meq} / \mathrm{L})\end{array}$ & $\underset{(\mu \mathrm{mol})}{\mathrm{SiO}_{2}}$ & $\begin{array}{l}\delta 18_{0} \\
(\% 0)^{\mathrm{a}}\end{array}$ \\
\hline $2 \mathrm{H}-3,120-125$ & 6.70 & 7.90 & 11.81 & 49.70 & 112 & 12.2 & 560 & 26.6 & 3.94 & 726 & -0.4 \\
\hline $5 \mathrm{H}-3,120-125$ & 35.40 & 7.94 & 16.53 & 41.11 & 125 & 9.3 & 567 & 17.3 & 3.84 & 1245 & -2.2 \\
\hline $8 \mathrm{H}-4,120-125$ & 65.70 & 8.21 & 19.28 & 36.13 & 123 & 12.4 & 571 & 12.0 & 4.40 & 826 & -2.7 \\
\hline $3 R-3,120-125$ & 90.40 & 8.23 & 18.32 & 37.54 & 123 & 10.4 & 560 & 15.3 & 3.73 & 892 & -2.0 \\
\hline $6 \mathrm{R}-3,120-125$ & 119.40 & 8.14 & 23.23 & 30.68 & 136 & 8.9 & 572 & 6.4 & 4.14 & 1020 & -3.5 \\
\hline $20 \mathrm{R}-2,120-125$ & 233.80 & 7.84 & 29.11 & 28.66 & 171 & 6.8 & 578 & 5.3 & 2.64 & 1417 & -3.5 \\
\hline $26 \mathrm{R}-2,120-125$ & 291.70 & 7.84 & 28.38 & 29.76 & 172 & 6.6 & 577 & 7.9 & 2.64 & 1154 & -2.4 \\
\hline $34 \mathrm{R}-1,120-125$ & 367.50 & 7.67 & 24.83 & 28.64 & 162 & 6.6 & 579 & 5.5 & 3.07 & 1449 & -2.9 \\
\hline $53 R-4,115-125$ & 554.55 & 8.34 & 37.17 & 20.46 & 168 & 6.1 & 577 & 0.1 & 2.65 & 423 & -3.9 \\
\hline $59 \mathrm{R}-3,115-125$ & 611.05 & 8.65 & 37.00 & 20.53 & 160 & 2.2 & 576 & 3.0 & 1.24 & 228 & -4.1 \\
\hline $62 \mathrm{R}-5,115-125$ & 651.96 & 8.57 & 40.14 & 20.43 & 167 & 2.1 & 583 & 2.4 & 1.79 & 308 & -4.0 \\
\hline
\end{tabular}

a Relative to SMOW.

\section{DISCUSSION}

At all sites depletion of pore water magnesium and enrichment of dissolved calcium relative to seawater is observed. At several sites the concentration profiles of calcium and magnesium are approximately linear, indicating that the pore water merely serve as a conduit between a deeper reaction zone and seawater. However, the ${ }^{87} \mathrm{Sr} /{ }^{86} \mathrm{Sr}$-ratios of the interstitial water strontium (Egeberg et al., this volume) indicate that reactions involving volcanic matter are taking place throughout the sediment column. Linear concentration gradients do not indicate that reactions are not taking place, but only that reactions consuming an element are stoichiometrically balanced by reactions producing the same element. Studies of $\mathrm{Rb}-\mathrm{Sr}$ systematics of alteration minerals in basalts drilled by DSDP indicate that interactions between seawater and layer II do not persist for more than 10-15 Ma after crust formation (Staudigel and Hart, 1985).
Of the sites included in this study, only Sites 689, 690 (Maud Rise), and Site 694 (Weddell abyssal plain) are underlain by oceanic crust. Sediments recovered at the Maud Rise indicate that the basement is of Cretaceous age, and seismic reflection data within the Weddell Basin suggest that it is of Mesozoic age (LaBrecque and Keller, 1982). Thus one would not expect the pore water composition to be influenced by seawater/crust interactions at any of the Leg 113 sites.

At all sites the alkalinity is considerably less than what one would expect from the observed depletion of sulfate (assuming a $1: 2$ stoichiometry of the bacterially mediated reaction, equation 1).

$$
2 \mathrm{CH}_{2} \mathrm{O}+\mathrm{SO}_{4}{ }^{2-}=\mathrm{H}_{2} \mathrm{~S}+2 \mathrm{HCO}_{3}{ }^{-}
$$

Saturation indices calculations at in-situ temperature employing a modified version of Wigley's (1977) speciation program, and 
Table 7. Interstitial water concentration data, Site 697.

\begin{tabular}{lrcccccccccc}
\hline $\begin{array}{c}\text { Core, section } \\
\text { interval }(\mathrm{cm})\end{array}$ & $\begin{array}{c}\text { Depth } \\
(\mathrm{mbsf})\end{array}$ & $\mathrm{pH}$ & $\begin{array}{c}\mathrm{Ca}^{2}+ \\
(\mathrm{mmol})\end{array}$ & $\begin{array}{c}\mathrm{Mg}^{2+} \\
(\mathrm{mmol})\end{array}$ & $\begin{array}{c}\mathrm{Sr}^{2}+ \\
(\mu \mathrm{mol})\end{array}$ & $\begin{array}{c}\mathrm{K}+ \\
(\mathrm{mmol})\end{array}$ & $\begin{array}{c}\mathrm{C} 1- \\
(\mathrm{mmol})\end{array}$ & $\begin{array}{c}\mathrm{SO}^{2-} \\
(\mathrm{mmol})\end{array}$ & $\begin{array}{c}\text { Alk. } \\
(\mathrm{meq} / \mathrm{L})\end{array}$ & $\begin{array}{c}\mathrm{SiO}_{2} \\
(\mu \mathrm{mol})\end{array}$ & $\begin{array}{r}\delta 18_{0} \\
(\%)^{\mathrm{a}}\end{array}$ \\
\hline $1 \mathrm{H}-4,145-150$ & 5.95 & 7.85 & 10.48 & 51.68 & 89 & 10.2 & 555 & 28.0 & 4.89 & 880 & -1.2 \\
$1 \mathrm{H}-1,145-150$ & 19.45 & 7.93 & 11.38 & 49.69 & 89 & 10.0 & 560 & 23.1 & 9.48 & 389 & -1.4 \\
$3 \mathrm{H}-4,120-125$ & 42.80 & 7.80 & 11.83 & 48.01 & 97 & 8.8 & 557 & 19.9 & 12.00 & 663 & -1.0 \\
$4 \mathrm{H}-4,115-125$ & 52.45 & 7.82 & 12.34 & 47.45 & 95 & 9.8 & 567 & 16.8 & 14.56 & 899 & -1.6 \\
$5 \mathrm{H}-3,115-125$ & 60.65 & & & & 89 & & & & & -1.5 \\
$6 \mathrm{H}-2,115-125$ & 68.85 & 7.94 & 12.66 & 45.17 & 90 & 9.2 & 567 & 13.1 & 16.64 & 761 & -1.0 \\
$7 \mathrm{H}-2,140-150$ & 78.90 & 7.89 & 12.90 & 44.48 & 93 & 7.9 & 561 & 13.1 & 16.11 & 884 & -2.5 \\
$8 \mathrm{H}-3,140-150$ & 90.10 & 8.10 & 13.42 & 43.15 & 93 & 8.3 & 566 & 10.3 & 17.53 & 926 & -3.3 \\
$9 \mathrm{H}-3,115-125$ & 99.55 & 8.06 & 13.43 & 42.86 & 93 & 7.4 & 565 & 10.3 & 17.35 & 909 & -1.4 \\
$13 \mathrm{X}-3,115-125$ & 132.75 & 7.78 & 14.01 & 40.25 & 102 & 5.4 & 566 & 6.5 & 18.35 & 1055 & -0.6 \\
$16 \mathrm{X}-4,115-125$ & 163.15 & 7.74 & 14.48 & 39.35 & 106 & 4.8 & 570 & 6.2 & 17.74 & 1129 & \\
$19 \mathrm{X}-3,115-125$ & 190.75 & 7.84 & 14.76 & 38.29 & 100 & 5.2 & 568 & 5.3 & 14.00 & 1117 & -0.9 \\
$22 \mathrm{X}-2,115-125$ & 218.15 & 7.99 & 14.87 & 35.99 & 103 & 5.8 & 569 & 5.0 & 13.77 & 1021 & -1.6 \\
$26 \mathrm{X}-2,115-125$ & 256.85 & 7.89 & 16.27 & 35.12 & 101 & 4.3 & 556 & 5.9 & 11.08 & 1020 & -1.8 \\
$29 \mathrm{X}-2,115-125$ & 285.90 & 7.93 & 16.21 & 35.71 & 79 & 2.8 & 671 & 4.7 & 11.57 & 1040 & \\
$31 \mathrm{X}-2,140-150$ & 305.50 & 8.27 & 17.89 & 32.44 & 111 & 2.9 & 566 & 7.0 & 10.07 & 968 & -2.2 \\
\hline & & & & & & & & & & &
\end{tabular}

a Relative to SMOW.

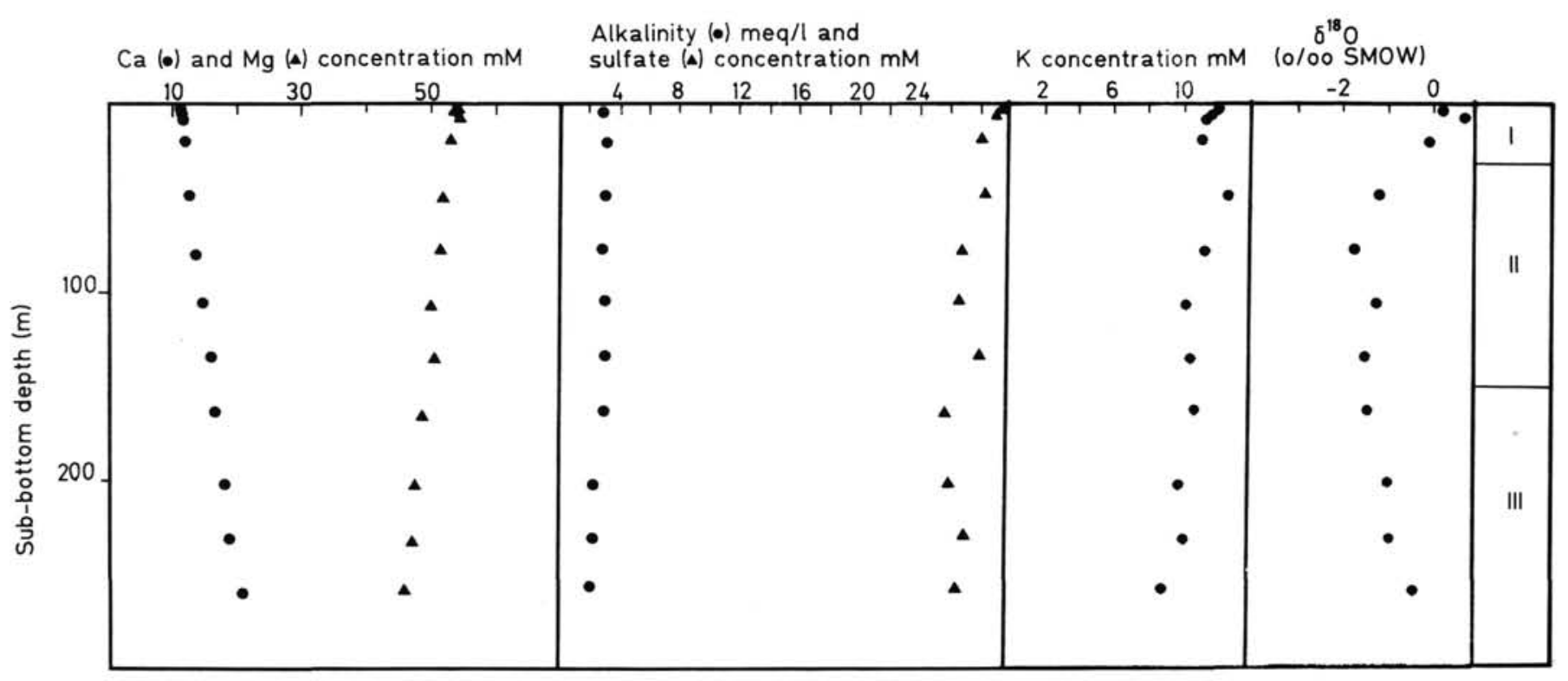

Figure 2. Concentration of calcium, magnesium, sulfate, alkalinity, and potassium and oxygen isotope distribution in pore water at Site 689, and lithostratigraphic boundaries. Data from Table 1.

the analytical results, reveal that all pore waters are saturated with respect to calcite. Thus, the alkalinity deficiency is probably caused by calcite precipitation. On a conventional $\mathrm{Mg}$ vs. $\mathrm{Ca}$ plot considerable scatter is observed (Fig. 9A). However, when the amount of calcium released is raised to account for the missing alkalinity (by adding half of the difference between the observed and the expected alkalinity to the observed calcium concentration), a far stronger correlation between magnesium and calcium is obtained (Fig. 9B). The slope of the best fit line in Figure 9B is -0.9 .

In seawater, dolomite is stable relative to calcite, and yet there is no evidence of recent dolomite formation in seawater of normal composition (Stumm and Morgan, 1981). Although dolomite is present in discrete layers at Sites 695 (111.95-112.1 mbsf), 696 (89.2-89.3 and 502.2-502.25 mbsf), and 697 (194.1200.7 mbsf) the average concentration of carbonate (less than $0.1 \%$ ) is too low for dolomitization to account for the observed depletion of magnesium and enrichment of calcium. Furthermore, because the fractionation factor for oxygen isotopes be- tween dolomite and calcite is close to 1 (Friedman and O'Neil, 1977), dolomitization can not account for the negative $\delta^{18} \mathrm{O}$ values of the pore water. Thus, while dolomitization may have been operative locally, additional mechanisms must be invoked to describe the overall chemical and isotope composition of the interstitial water.

An alternative mechanism that has gained much acceptance (e.g., Perry et al., 1976) involves alteration of volcanic matter. Visual inspection of the cores (Barker, Kennett, et al., 1988) revealed the presence of numerous pale greenish laminae interpreted as devitrified volcanics. The greenish color is usually attributed to the presence of authigenic smectite (Gardner et al., 1986). Also, at several sites, volcanic rock fragments were abundant.

Perry et al. (1976) concluded that alteration of calc-alkalic volcanics dispersed in the sediment, or basalt to a smectite phase, is responsible for the calcium and magnesium gradients and the depletion of pore water ${ }^{18} \mathrm{O}$. Based on a compilation of data given by Nelson et al. (1986), the average composition of 


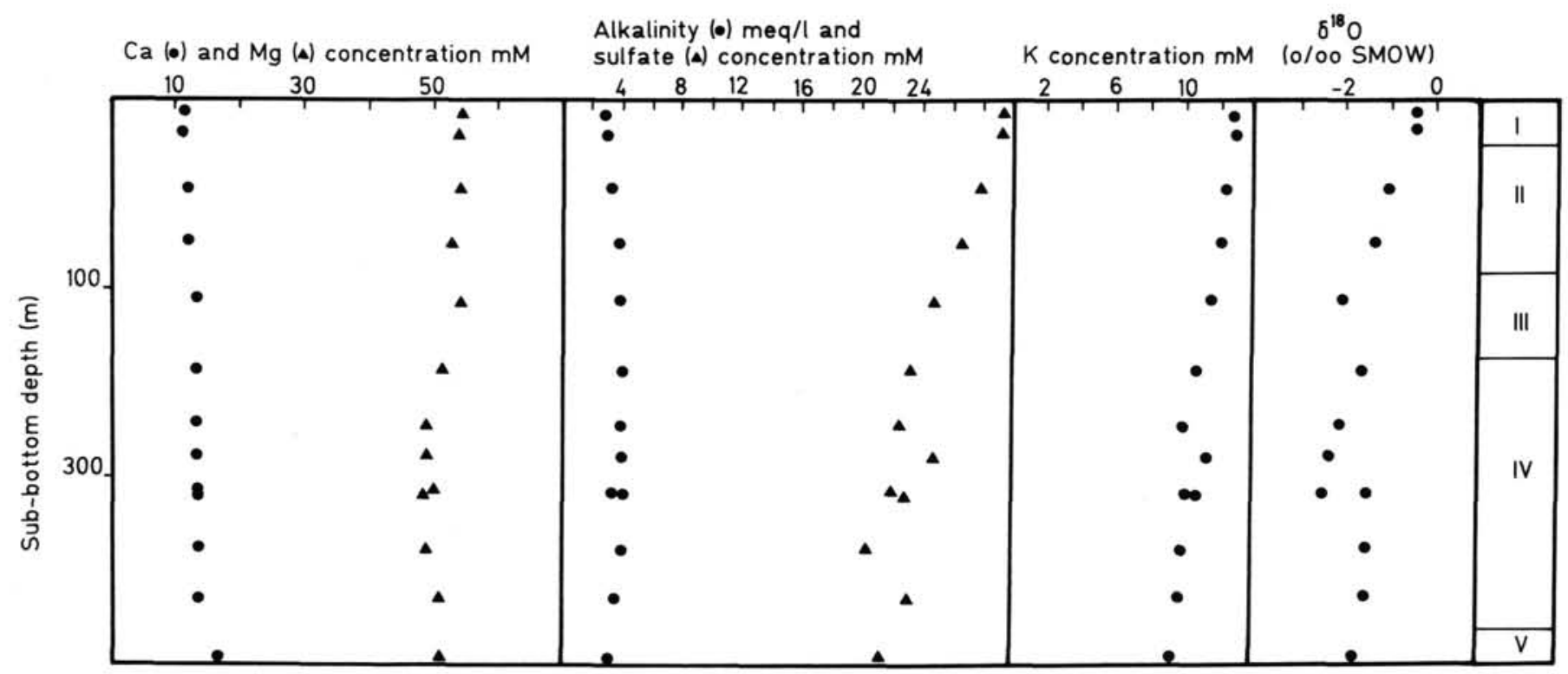

Figure 3. Concentration of calcium, magnesium, sulfate, alkalinity, and potassium and oxygen isotope distribution in pore water at Site 690 , and lithostratigraphic boundaries. Data from Table 2.

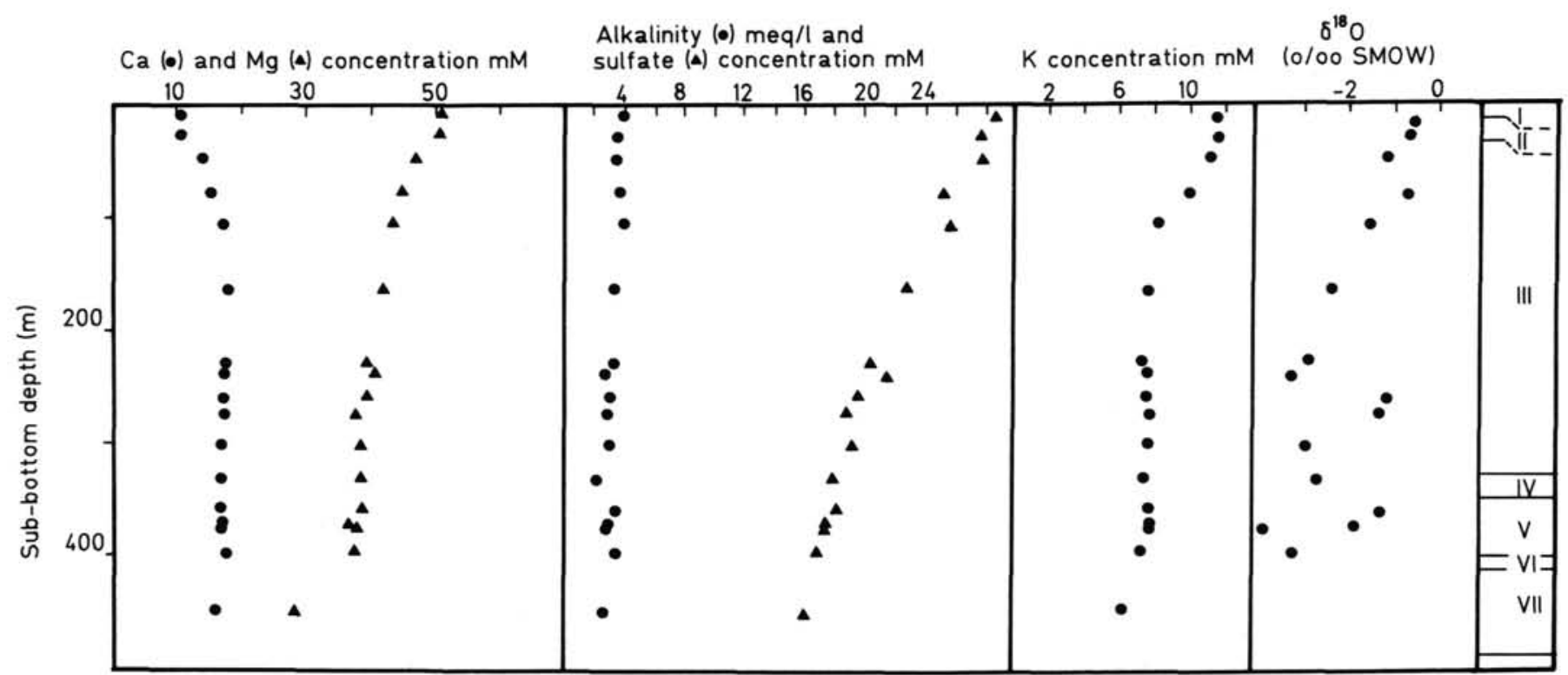

Figure 4. Concentration of calcium, magnesium, sulfate, alkalinity, and potassium and oxygen isotope distribution in pore water at Site 693, and lithostratigraphic boundaries. Data from Table 3.

basaltic glasses from mid-ocean ridges may be represented by the formula:

$$
\mathrm{Si}_{50} \mathrm{Al}_{10} \mathrm{Fe}_{5} \mathrm{Ca}_{7} \mathrm{Mg}_{6} \mathrm{Na}_{3} \mathrm{KO}_{135}
$$

Thus, the reaction of calc-alkalic volcanic debris to a threeoctahedral smectite (Drever, 1976), balanced only to account for the 1:1 relationship between calcium and magnesium, may be written:

$$
\begin{gathered}
7 \mathrm{Si}_{50} \mathrm{Al}_{10} \mathrm{Fe}_{5} \mathrm{Ca}_{7} \mathrm{Mg}_{6} \mathrm{Na}_{3} \mathrm{KO}_{135}+49 \mathrm{Mg}^{2+} \\
13 \mathrm{Si}_{6} \mathrm{O}_{20}(\mathrm{OH})_{4} \mathrm{Al}_{2} \mathrm{Mg}_{7}+49 \mathrm{Ca}^{2+}
\end{gathered}
$$

Inspection of equation 3 reveals that because of the much higher $\mathrm{Al} / \mathrm{Mg}$ and $\mathrm{Si} / \mathrm{Mg}$ ratio of the volcanic glass, the reaction liberates significant amounts of aluminum and silica. Because aluminum is essentially immobile, the reaction is probably accompanied by the precipitation of other aluminous authigenic phases. Based on the extensive occurrence of zeolites in deep sea sediments and the ubiquitous association between volcanic glass and phillipsite (Stonecipher, 1977; Kastner and Stonecipher, 1978), we balance the reaction with phillipsite. This also provides a sink for potassium. In accordance with petrographic observations, the excess silicon is precipitated as chert, and ferrous iron as sulfide. Thus the complete reaction may be written: 


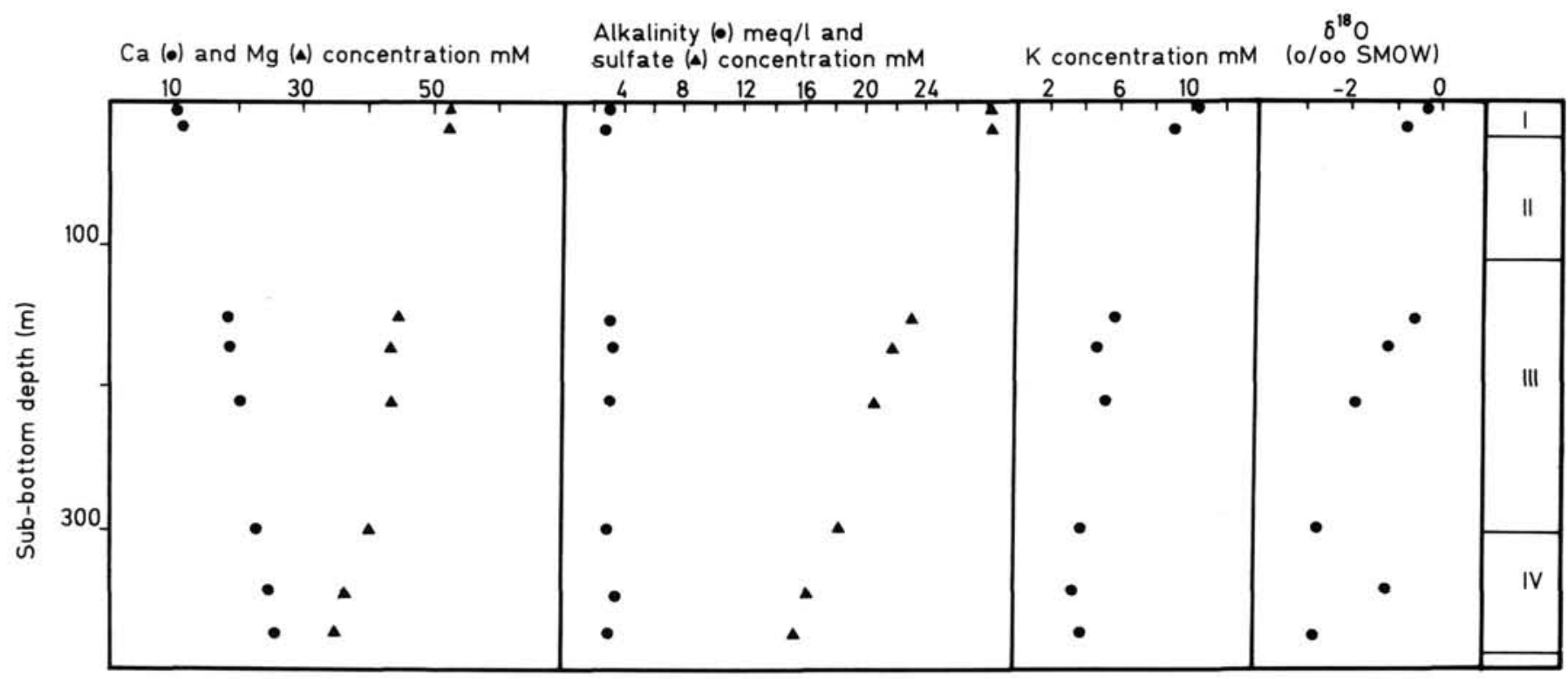

Figure 5. Concentration of calcium, magnesium, sulfate, alkalinity, and potassium and oxygen isotope distribution in pore water at Site 694, and lithostratigraphic boundaries. Data from Table 4.

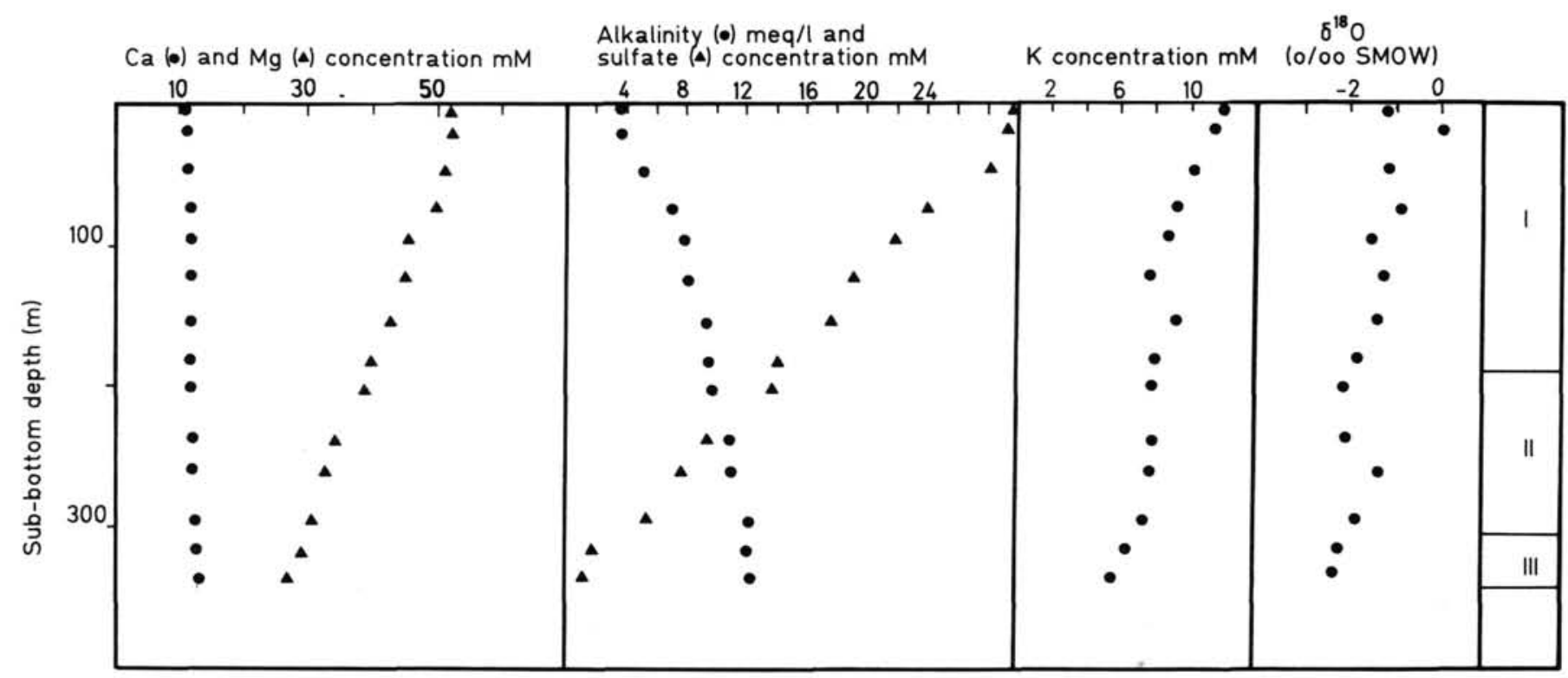

Figure 6. Concentration of calcium, magnesium, sulfate, alkalinity, and potassium and oxygen isotope distribution in pore water at Site 695, and lithostratigraphic boundaries. Data from Table 5.

$$
\begin{gathered}
7 \mathrm{Si}_{50} \mathrm{Al}_{10} \mathrm{Fe}_{5} \mathrm{Ca}_{7} \mathrm{Mg}_{6} \mathrm{Na}_{3} \mathrm{KO}_{135}+49 \mathrm{Mg}^{2+}+16 \mathrm{~K}^{+}+109 \mathrm{H}_{2} \mathrm{O} \\
+54 \mathrm{H}+35 \mathrm{~S}^{2-}=>13 \mathrm{Si}_{6} \mathrm{O}_{20}(\mathrm{OH})_{4} \mathrm{Al}_{2} \mathrm{Mg}_{7} \\
+49 \mathrm{Ca}^{2+}+22\left(\mathrm{Na}_{\mathrm{x}} \mathrm{K}_{1-\mathrm{x}}\right)_{2} \mathrm{Al}_{2} \mathrm{Si}_{5} \mathrm{O}_{14} \\
\cdot 5 \mathrm{H}_{2} \mathrm{O}+16 \mathrm{SiO}_{2}+35 \mathrm{FeS}
\end{gathered}
$$

$\mathrm{x}$ is 0.48 .

Including phillipsite and chert in the reaction has a very strong effect on the ${ }^{18} \mathrm{O} /{ }^{16} \mathrm{O}$-budget. In order to obtain mass balance for the oxygen isotopes, Perry et al.,(1976) reacted volcanic glass to produce 25 vo $1 . \%$ authigenic smectite. Lawrence et al. (1975) postulated that interactions between seawater and layer II basalts could account for the depletion of pore water ${ }^{18} \mathrm{O}$. However, by using the stoichiometry of equation 4 and the partition coefficients between water and minerals listed in Table 8 , it is estimated that at $4^{\circ} \mathrm{C}$ alteration of $5 \mathrm{vol} . \%$ calc-alkalic volcanics will shift the pore water to $-2.2 \%$ (SMOW, Standard Mean Ocean Water). The alteration of $10 \%$ will take the pore water to $-4 \%$ (Fig. 10). This produces 1.5 and 3 vo1. $\%$ authigenic smectite and 1.9 and 3.8 vol. $\%$ zeolites, respectively (at $50 \%$ porosity). Figure 10 shows that within the expected temperature range, the equilibrium ${ }^{18} \mathrm{O}$ distribution is not very sensitive to the assumed temperature. For the above calculations, the fractionation of oxygen isotopes between pore water and the crystal water of phillipsite is neglected. For calculations at temperatures higher than $4^{\circ} \mathrm{C}$, we follow the approach taken by Lee (1987) who (on the basis of compositional and structural simi- 


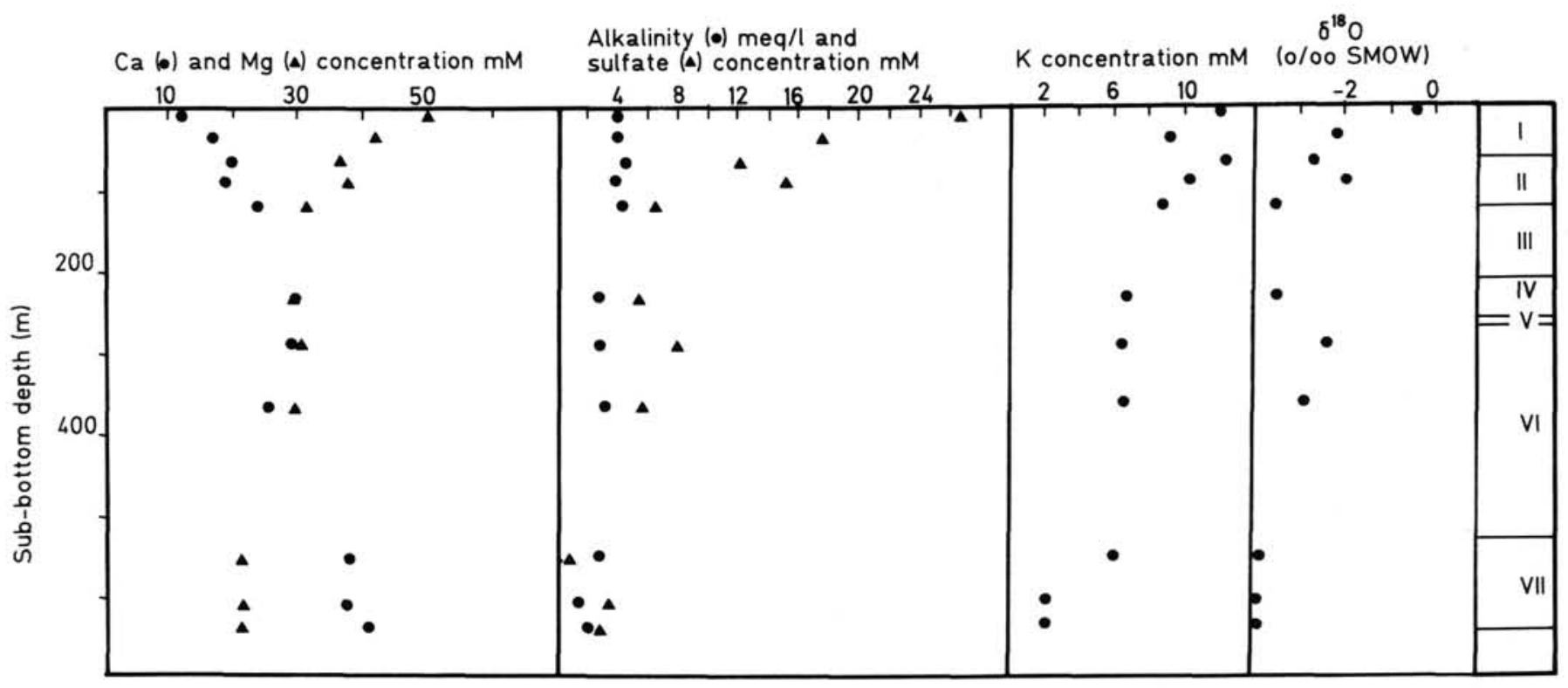

Figure 7. Concentration of calcium, magnesium, sulfate, alkalinity, and potassium and oxygen isotope distribution in pore water at Site 696, and lithostratigraphic boundaries. Data from Table 6.

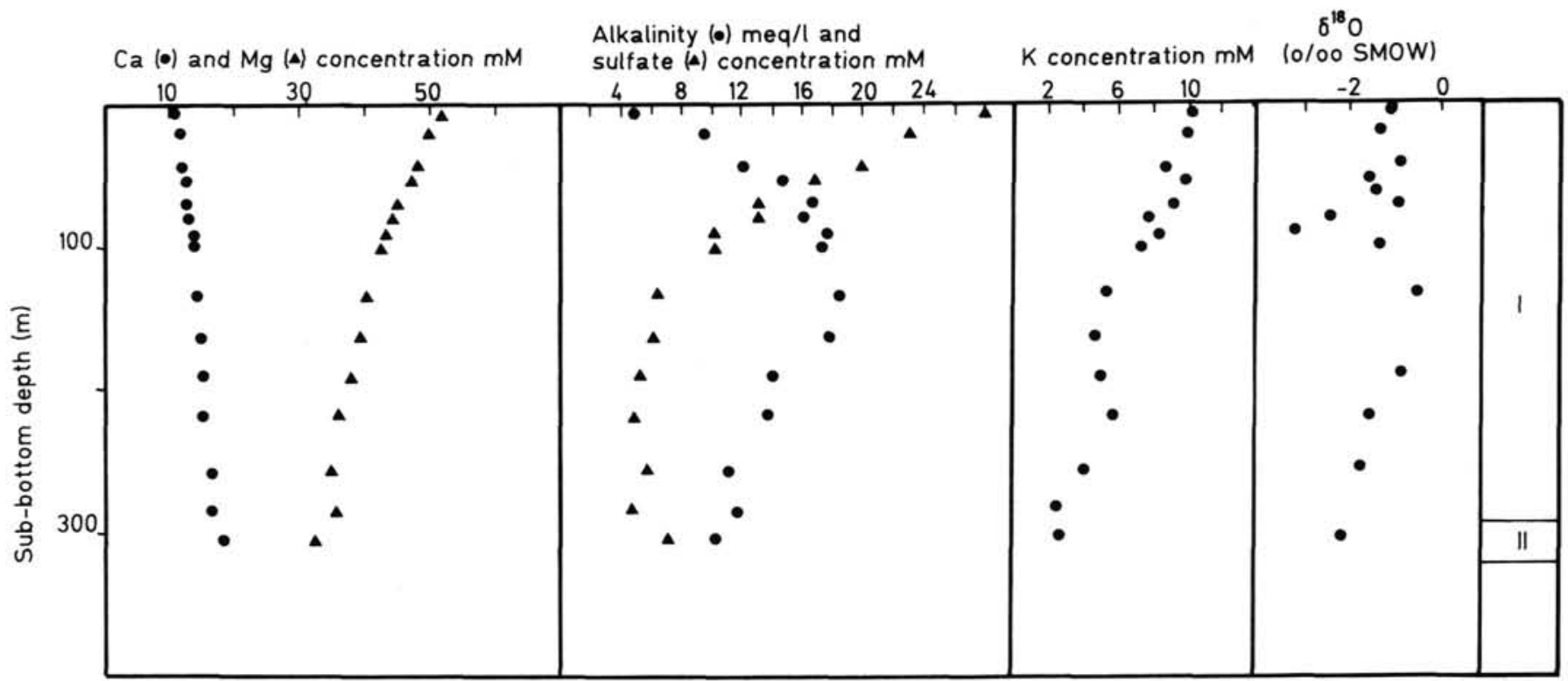

Figure 8. Concentration of calcium, magnesium, sulfate, alkalinity, and potassium and oxygen isotope distribution in pore water at Site 697, and lithostratigraphic boundaries. Data from Table 7.

larities) substituted the temperature dependent fractionation factor of alkali feldspar for zeolite. The $\delta^{18} \mathrm{O}$ values of the volcanic glass and initial pore water is assumed to be $+8 \%$ (SMOW) (Taylor, 1968) and 0\% (SMOW), respectively. The equilibrium oxygen isotope distribution was estimated by the means of equation 5 .

$$
\delta^{18} \mathrm{O}=1000 \cdot\left(\mathrm{R}_{\mathrm{w}}-\mathrm{R}_{\mathrm{SMOW}}\right) / \mathrm{R}_{\mathrm{SMOW}}
$$

where $\mathrm{R}_{\mathrm{w}}$ and $\mathrm{R}_{\mathrm{SMOw}}$ are the ${ }^{18} \mathrm{O} /{ }^{16} 0$ ratios of the interstitial water at equilibrium and of Standard Mean Ocean Water, respectively. $R_{w}$ estimated by the means of equation 6 .

$$
\begin{aligned}
\mathrm{R}_{\mathrm{w}}= & \left(\mathrm{N}_{\mathrm{G}} \cdot(1-\mathrm{P}) \cdot \mathrm{R}_{\mathrm{G}} \cdot \mathrm{S}_{\mathrm{G}} \cdot \text { vol.\% }+\mathrm{P} \cdot \mathrm{R}_{\mathrm{w}}{ }^{\circ} \cdot \mathrm{S}_{\mathrm{G}} \cdot \mathrm{V}_{\mathrm{G}}\right. \\
& \left.\cdot 100 / \mathrm{M}_{\mathrm{w}}\right) /\left(\mathrm{P} \cdot \mathrm{S}_{\mathrm{G}} \cdot \mathrm{V}_{\mathrm{G}} \cdot 100 / \mathrm{M}_{\mathrm{w}}\right. \\
& +(1-\mathrm{P}) \cdot\left(1+\Sigma \mathrm{S}_{\mathrm{i}} \cdot \mathrm{N}_{\mathrm{i}} \cdot \alpha_{\mathrm{i}}\right) \cdot \text { vol. \%) }
\end{aligned}
$$

where the subscript $\mathrm{G}$ denotes glass and $\mathrm{i}$ the various products, $\mathrm{P}$ is the porosity (fraction), $\mathrm{R}_{\mathrm{G}}$ and $\mathrm{R}_{\mathrm{w}}{ }^{\circ}$ are the ${ }^{18} \mathrm{O} /{ }^{16} \mathrm{O}$ ratios of glass and initial seawater, respectively. $\mathrm{V}_{\mathrm{G}}$ is the molar volume of glass, $S_{i}$ refers to the stoichiometric coefficients in the reaction (equation 4 ), $N_{i}$ is the number of moles of oxygen in each unit of minerals, $\alpha_{\mathrm{i}}$ is the oxygen isotope fractionation factor between mineral $i$ and water, $\mathbf{M}_{w}$ is the molar weight of water and vol. $\%$ is the percentage of volcanic matter reacted. The 


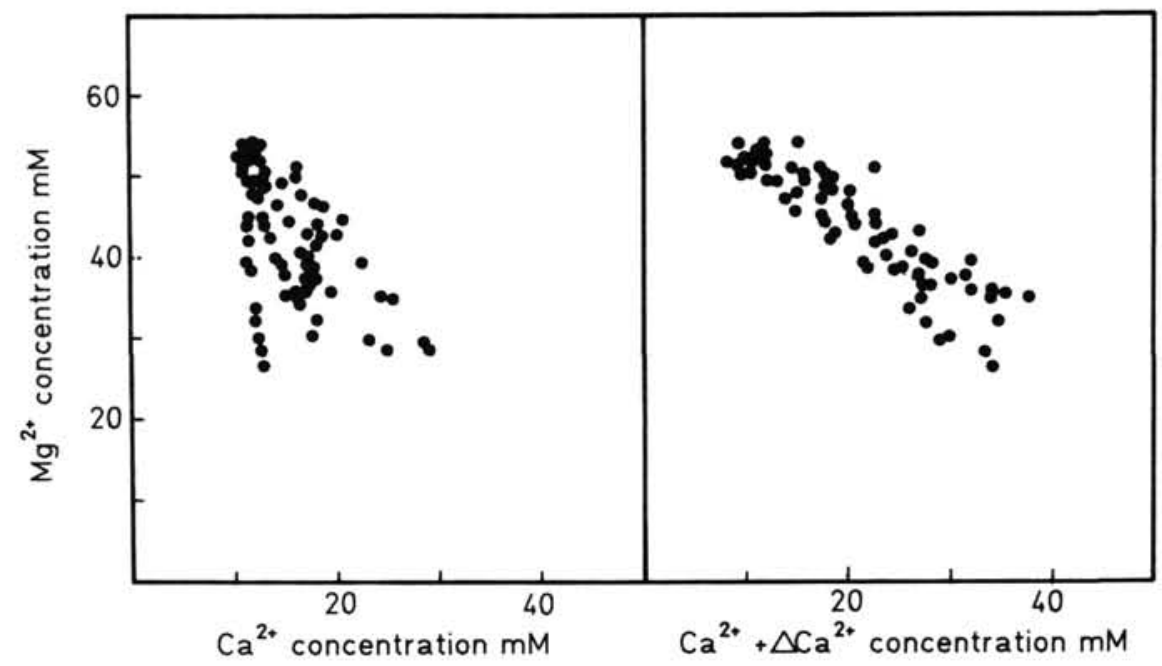

Figure 9. A. Concentration of magnesium vs. concentration of calcium. B. Concentration of magnesium vs. concentration of calcium corrected for calcite precipitation.

Table 8. Fractionation factors between minerals and water. See text for discussion.

\begin{tabular}{|c|c|c|c|}
\hline Mineral & $\begin{array}{l}1000 \operatorname{Ln} \alpha \\
\left(4^{\circ} \mathrm{C}\right)\end{array}$ & $1000 \underset{(\mathrm{f}(\mathrm{t}))}{\operatorname{Ln} \alpha}=\mathrm{f}(\mathrm{t})$ & Reference \\
\hline Smectite & 29.9 & $2.67 \cdot 10^{6} \cdot T^{-2}-4.82$ & Yeh and Savin (1976) \\
\hline Chert & 36.9 & $3.09 \cdot 10^{6} \cdot T-2-3.29$ & Knauth and Epstein (1976) \\
\hline Zeolite & 33.4 & & Savin and Epstein (1970) \\
\hline Feldspar & 34.3 & $2.90 \cdot 10^{6} \cdot \mathrm{T}^{-2}-3.41$ & O'Neil and Taylor (1967) \\
\hline
\end{tabular}

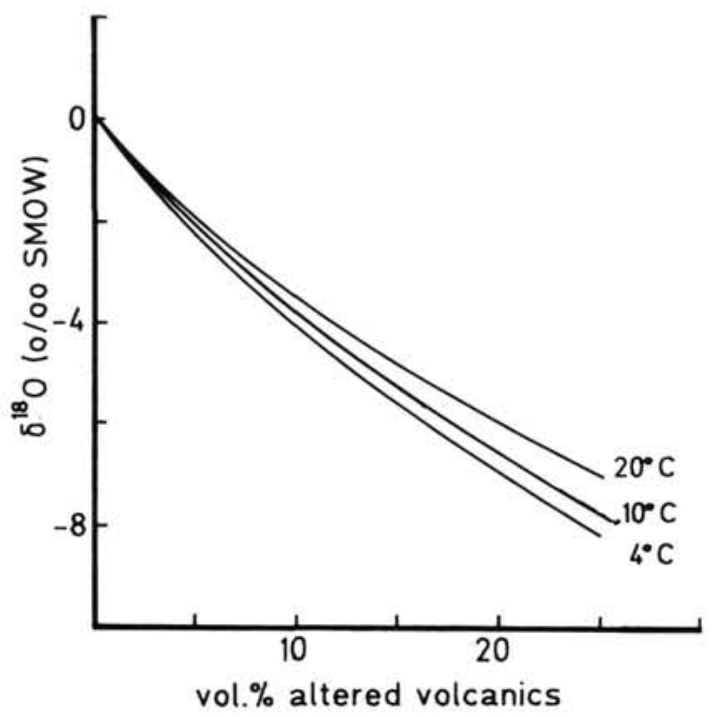

Figure 10. Alteration of basic volcanic material: effect on pore water oxygen isotope distribution calculated by the means of equations 5 and 6 .

summation in the denominator is performed over all the products.

The effect of alteration of volcanic matter on the $\delta^{18} \mathrm{O}$ values of the pore water found in this study is much stronger than recognized in previous studies (Lawrence et al, 1975; Perry et al., 1976) because we consider the complete reaction. The significance of this finding is that the pore water may evolve their negative $\delta^{18} \mathrm{O}$ values without invoking an external source or unrea- sonably large amounts of volcanic debris. Furthermore, as 5 vol. $\%$ calc-alkalic glass only constitutes about $15 \mathrm{mmol} / \mathrm{L}$ of sediment (estimated based on the formula for volcanic glass given above, a density of $2.5 \mathrm{~g} / \mathrm{cm}^{3}$ and $50 \%$ porosity), there is little doubt that diffusion can supply the amount of magnesium and potassium consumed. However, mathematical verification of this statement would involve treatment of multicomponent diffusion and is beyond the scope of this presentation.

At first glance equation 4 may seem somewhat ad hoc, however it is in accordance with petrographic observations and accounts for the isotope ratio of the pore water and the general features of the major element chemistry. Calcium and magnesium react in a 1:1 fashion (after corrections for the missing alkalinity have been done), sodium is preserved, and potassium decreases. Inspection of Figures 11 and 12 reveals that the observed $\mathrm{K} / \mathrm{Mg}$ ratio is fairly close to the ratio predicted by the reaction (16/49 mole/mole), and that the relationship between the concentration of magnesium and the $\delta^{18} \mathrm{O}$ values of the pore wa-

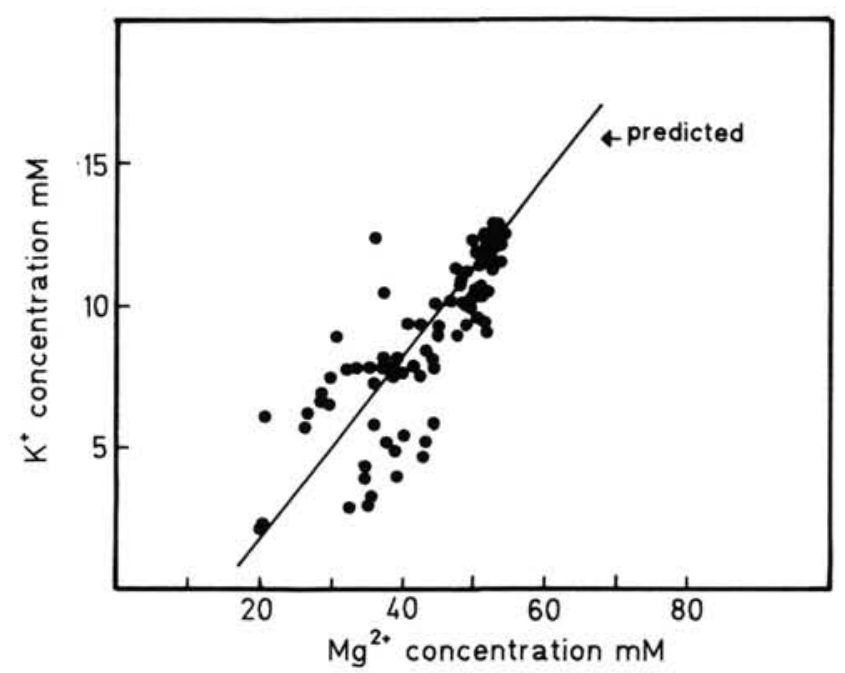

Figure 11. Concentration of potassium vs. concentration of magnesium compared to the slope given by the ratio of the stoichiometric coefficient in equation 4. 


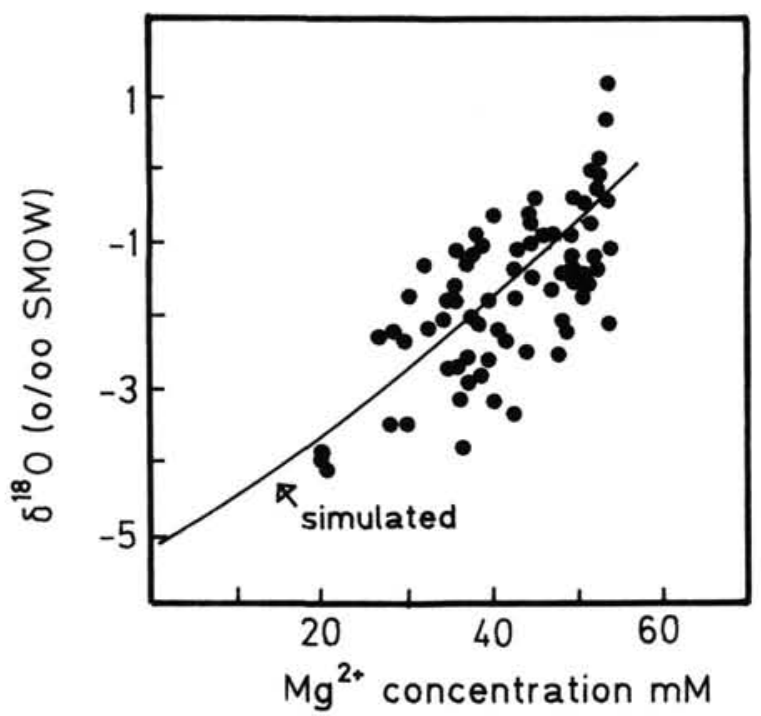

Figure 12. Oxygen isotope distribution vs. concentration of magnesium compared to the model output calculated by transforming from vol. $\%$ volcanic material in equation 6 to concentration of magnesium by the means of the stoichiometry of equation 4 .

ter follows the predicted trend. Much of the scatter in Figures 11 and 12 is attributed to compositional variations of reactants and products. For example, charge balance calculations indicated that to a variable degree sodium is removed from the pore water, thus the relative amount of sodium and potassium that is incorporated into zeolites may deviate from the ratio proposed by the stoichiometry of reaction 4 . Figures $2-8$ show that none of the observed variations in pore water composition can be directly related to lithostratigraphic variations. The much lower gradients of interstitial constituents at the pelagic sites (Sites 689 and 690 ) could equally well be due to lower rate of sedimentation as to less-abundant volcanogenic material. The absence of any correlation between bulk sediment composition and pore water composition indicates that the concentration of dissolved components is controlled by a minor phase.

The hypothesis put forward above should be tested either by measuring the $\delta^{18} \mathrm{O}$ values of the alteration products or by detailed studies of the relative abundance of reactants and products. It was shown above that even the most negative pore water $\delta^{18} \mathrm{O}$ value encountered in this study could have been caused by alteration of less than about $10 \mathrm{vol} . \%$ volcanics. This would result in about 3 vol. $\%$ smectite and 3.8 vol. $\%$ zeolite. Separation of these small amounts for oxygen isotope analyses from a complex matrix of hemipelagic mud is a formidable task. Because of variable composition at time of deposition, and the problems of distinguishing between detrital and authigenic phases, unambiguous variations in the relative abundance of reactants and products are difficult to detect. Most probably, detailed studies of thick ash layers offer the best opportunity to reject or verify the hypothesis.

\section{CONCLUSIONS}

The absence of any correlation between bulk sediment composition and pore water composition indicates that the concentration of interstitial water constituents is controlled by a minor phase. Petrographic observations and general geochemical considerations indicate that reactions involving alteration of calcalkalic volcanic material are an important process. Mass balance estimates show that most of the variations in major ele- ment chemistry and isotope composition of Leg 113 interstitial water may be interpreted in terms of alteration of volcanic matter. Based on the extensive occurrence of zeolites in deep sea sediments and the ubiquitous association between volcanic glass, smectite, phillipsite, chert, and pyrite, a diagenetic reaction was constructed. The diagenetic reaction accounts for the $1: 1$ relationship between $\mathrm{Ca}$ and $\mathrm{Mg}$ and the depletion of potassium. Mass balance calculations taking the effect of zeolites and chert into consideration reveal that the pore water may evolve their negative $\delta^{18} \mathrm{O}$ values without having to invoke external sources or unreasonably large amounts of volcanic matter. The pore waters may have evolved their chemical and isotope composition through alteration of less than $10 \mathrm{vol} . \%$ volcanic glass. Alteration of this amount of volcanogenic material would produce less than 4 vol. \% authigenic smectite and phillipsite. Thus, it is difficult to verify the hypothesis by quantitative petrographic observations.

\section{ACKNOWLEDGMENTS}

Financial support from the Norwegian Research Council for Sciences and the Humanities and from the Norwegian Academy of Science and Letters is gratefully acknowledged.

\section{REFERENCES}

Baker, P. A., 1986. Pore-water chemistry of carbonate-rich sediments, Lord Howe Rise, Southwest Pacific Ocean. In Kennett, J. P., von der Borch, C.C, Baker, P. A., et al., Init. Repts. DSDP, 90: Washington (U.S. Govt. Printing Office), 1249-1256.

Barker, P. F., Kennett, J. P., et al., 1988. Proc., ODP, Init. Repts., 113: College Station, TX (Ocean Drilling Program).

Drever, J. I., 1971. Magnesium-iron replacement in clay minerals in anoxic marine sediments. Science 172:1334-1336.

, 1974. The magnesium problem. In Goldberg, E. D. (Ed.), The Sea (Vol.5): New York (Wiley-Interscience), 337-357.

1976. Chemical and mineralogical studies, Site 323. In Hollister, C. D., Craddock, C., Bogdanov, Y. A., et al., Init. Repts. DSDP, 35: Washington (U.S. Printing Office), 471-477.

Gardner, J. V., Nelson, C. S., and Baker, P. A., 1986. Distribution and character of pale green laminae in sediments from Lord Howe Rise: A probable late Neogene and Quaternary tephrostratigraphic record. In Kennett, J. P., von der Borch, C. C., Baker, P. A., et al., Init. Repts. DSDP, 90: Washington (U.S. Govt. Printing Office), 11451159.

Gieskes, J. M., 1973. Interstitial water studies, Leg 15-Alkalinity, pH, $\mathrm{Mg}, \mathrm{Ca}, \mathrm{Si}, \mathrm{PO}_{4}$, and $\mathrm{NH}_{4}$. In Heezen, B. C., MacGregor, I. D., Foreman, H. P., et al., Init. Repts. DSDP, 20: Washington (U.S. Govt. Printing Office), 813-829.

1974. Interstitial water studies. Leg 25. In Simpson, E.S.W., Schlich, R., Gieskes, J. M., et al., Init. Repts. DSDP, 25: Washington (U.S. Govt. Printing Office), 361-394.

Gieskes, J. M., and Lawrence, J. R., 1976. Interstitial water studies, Leg 35. In Hollister, C. D., Craddock, C., Bogdanov, Y. A., et al., Init. Repts. DSDP, 35: Washington (U.S. Govt. Printing Office), 407424.

Gieskes, J. M., Lawrence, J. R., and Galleisky, G., 1978. Interstitial water studies Leg 38. In Talwani, M., Udintsev, G., Bjorklund, K. B., et al., Init. Repts. DSDP, Suppl. to Vols. 38, 39, 40, and 41: Washington (U.S. Govt. Printing Office), 121-133.

Gieskes, J. M., Elderfield, H., Lawrence, J. R., and Lakind, J., 1984. Interstitial water studies Leg 78A. In Biju-Duval, B., Moore, J. C., Bergen, J. A. et al., Init. Repts. DSDP, 78A and 78B: Washington (U.S. Govt. Printing Office), 377-384.

Hawkesworth, C. J., and Elderfield, H., 1978. The strontium isotope composition of interstitial waters from sites 245 and 336 of the Deep Sea Drilling Project. Earth Planet. Sci. Lett., 40:423-432.

Heller-Kallai L., and Rozenson, I., 1978. Removal of magnesium from interstitial waters in reducing environments-the problem reconsidered. Geochim. Cosmochim. Acta, 42:1907-1909.

Kastner, M., and Stonecipher, S. A., 1978. Zeolites in pelagic sediments of the Atlantic, Pacific and Indian Oceans. In Sand, L. B. and 
Mumpton, F. A. (Eds.), Natural zeolites, occurrence, properties, use: Oxford (Pergamon Press), 199- 220.

Knauth, L. P., and Epstein, S., 1976. Hydrogen and oxygen isotope ratios in nodular and bedded cherts. Geochim. Cosmochim. Acta, 40: 1095-1108.

LaBrecque, J. L., and Keller, M., 1982. A geophysical study of the Indo-Atlantic Basin. In Craddock, C. (Ed.), Antarctic Geoscience: Madison, WI (Univ. Wisconsin Press), 387-396

Lawrence, J. R., Gieskes, J. M., and Broecker, W. S., 1975. Oxygen isotope and cation composition of DSDP pore waters and the alteration of layer II basalts. Earth Planet. Sci. Lett., 27:1-10.

Lee, Y. I., 1987. Isotopic aspects of thermal and burial diagenesis of sandstones at DSDP Site 445, Daito Rise, Northwest Pacific Ocean. Chem. Geol., 65: 95-102.

Mackenzie, F. T., and Garrels, R. M., 1966. Chemical mass balance between rivers and Oceans. Am. J. Sci., 264:507-525.

Manheim, F. T., 1976. Interstitial waters of marine sediments. In Riley, J. P. and Chester, R. (Eds.), Chemical Oceanography (Vol.6): London (Academic Press), 115-186.

Manheim, F. T., and Sayles, F. L., 1974. Composition and origin of interstitial waters of marine sediments based on deep sea drill cores. In Goldberg, E. D. (Ed.), The Sea (Vol. 5): New York (Wiley-Interscience), 527-568.

Matter A., Douglas R. G., and Perch-Nielsen, K., 1975. Fossil preservation, geochemistry, and diagenesis of pelagic carbonates from the Shatsky Rise, Northwest Pacific. In Larson, R. L., Moberly, R., Bukry, D., et al., Init. Repts. DSDP, 32: Washington (U.S. Govt. Printing Office), 891-921.

McDuff, E., 1981. Major cation gradients in DSDP interstitial waters: the role of diffusive exchange between sea water and upper oceanic crust. Geochim. Cosmochim. Acta, 34:105-120.

Nelson, P. C., Froggatt, P. C., and Gosson, G. J., 1986. Nature, chemistry and origin of late Cenozoic megascopic tephras in Leg 90 cores
Baker, P. A., et al., Init. Repts. DSDP, 90: Washington (U.S. Govt. Printing Office), 1161-1171.

O' Neil, J. R., and Taylor, H. P., 1967. The oxygen isotope and cation exchange chemistry of feldspars. Am. Mineral., 52:1414-1437.

Perry, E. A., Gieskes, J. M., and Lawrence, J. R., 1976. Mg, Ca and $\mathrm{O}^{18} / \mathrm{O}^{16}$ exchange in the sediment-pore water system, Hole 149 , DSDP. Geochim. Cosmochim. Acta, 40:413-423

Savin, S. M., and Epstein, S., 1970. The oxygen and hydrogen-isotope geochemistry of ocean sediments and shales. Geochim. Cosmochim. Acta, 34:43-63.

Staudigel, H., and Hart, S. R., 1985. Dating of ocean crust hydrothermal alteration: strontium isotope ratios from Hole 504B and a reinterpretation of $\mathrm{Sr}$ isotope data from Deep Sea Drilling Project Sites 105, 232, 417 and 418. In Anderson, R. N., Honnorez, J., Becker, K., et al., Init. Repts. DSDP, 83: 297-303.

Stonecipher, S. A., 1977. Origin, distribution and diagenesis of deep-sea clinoptilolite and phillipsite (Ph.D. dissert.). Scripps Inst. Oceanography.

Stumm, W., and Morgan, J. J., 1981. Aquatic Chemistry. New York (Wiley).

Taylor, H. P., 1968. The oxygen isotope geochemistry of igneous rocks. Contr. Mineral. Petrol., 19:1-17.

Wigley, T.M.L., 1977. WATSPEC: a computer program for determining the equilibrium speciation of aqueous solutions. Br. Geomorph. Res. Group, Tech. Bull., 20:1-40.

Wollast, R., 1974. The silica problem. In Goldberg, E. D. (Ed.), The Sea (Vol. 5): London (Wiley-Interscience), 359-392.

Yeh, H. W., and Savin, S. M., 1976. The extent of oxygen isotope exchange between clay minerals and sea water. Geochim. Cosmochim. Acta, 40:743-748.

Date of initial receipt: 12 January 1988

Date of acceptance: 17 November 1988

Ms 113B-169 